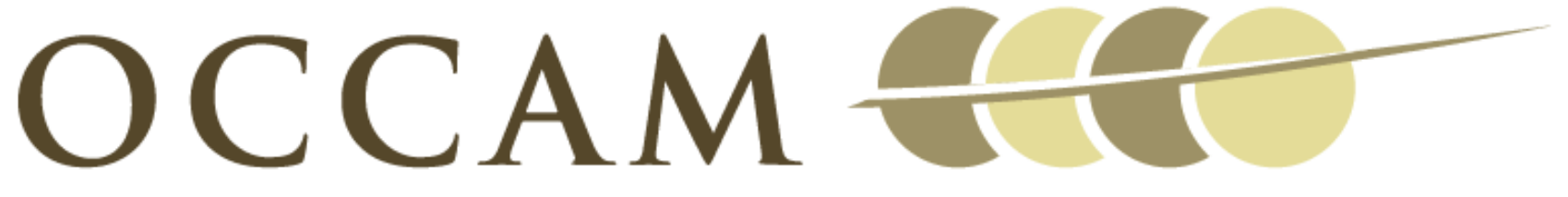

OXFORD CENTRE FOR COLLABORATIVE APPLIED MATHEMATICS

Report Number 10/15

Travelling waves in hyperbolic chemotaxis equations by

Chuan Xue, Hyung Ju Hwang, Kevin J Painter, Radek Erban

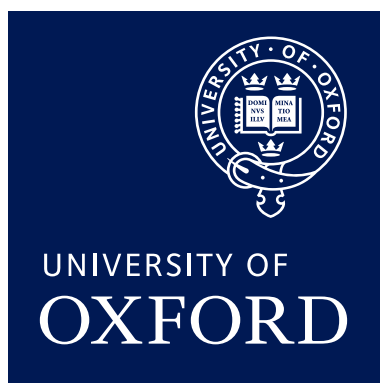

Oxford Centre for Collaborative Applied Mathematics Mathematical Institute 24 - 29 St Giles'

Oxford

OX1 3LB

England 

Bulletin of Mathematical Biology manuscript No.

(will be inserted by the editor)

\title{
Travelling waves in hyperbolic chemotaxis equations
}

\author{
Chuan Xue ${ }^{1}$, Hyung Ju Hwang ${ }^{2}$, Kevin J Painter ${ }^{3}$, Radek Erban ${ }^{4}$ \\ 1 Mathematical Biosciences Institute, Ohio State University, Jennings Hall 3rd Floor, 1735 Neil Ave., Columbus, OH \\ 43210, USA; e-mail: cxue@mbi.osu.edu \\ 2 Department of Mathematics, Pohang University of Science and Technology, Pohang 790-784, Republic of Korea; \\ e-mail: hjhwang@postech.ac.kr \\ 3 Department of Mathematics and Maxwell Institute for Mathematical Sciences, Heriot-Watt University, Edinburgh \\ EH14 4AS, United Kingdom; e-mail: painter@ma.hw.ac.uk \\ 4 Mathematical Institute, University of Oxford, 24-29 St Giles', Oxford, OX1 3LB, United Kingdom; e-mail: er- \\ ban@maths.ox.ac.uk
}

Received: date / Revised version: date

\begin{abstract}
Mathematical models of bacterial populations are often written as systems of partial differential equations for the densities of bacteria and concentrations of extracellular (signal) chemicals. This approach has been employed since the seminal work of Keller and Segel in the 1970s [Keller and Segel, J. Theor. Biol., 1971]. The system has been shown to permit travelling wave solutions which correspond to travelling band formation in bacterial colonies, yet only under specific criteria, such as a singularity in the chemotactic sensitivity function as the signal approaches zero. Such a singularity generates infinite macroscopic velocities which are biologically unrealistic. In this paper, we formulate a model that takes into consideration relevant details of the intracellular processes while avoiding the singularity in the chemotactic sensitivity. We prove the global existence of solutions and then show the existence of travelling wave solutions both numerically and analytically.
\end{abstract}

Key words travelling wave - velocity jump process - chemotaxis

\section{Introduction}

In 1966, Adler [2-4] observed the formation of travelling bands of motile bacteria, attributed to the chemotactic response of cells to oxygen and energy sources such as galactose, glucose, aspartate, threonine or serine. A phenomenological theory for these travelling bands was initiated by Keller and Segel [23], based on the following system of partial differential equations (PDEs):

$$
\begin{aligned}
& \frac{\partial n}{\partial t}=D_{n} \frac{\partial^{2} n}{\partial x^{2}}-\frac{\partial}{\partial x}\left[n \chi(S) \frac{\partial S}{\partial x}\right], \\
& \frac{\partial S}{\partial t}=D_{S} \frac{\partial^{2} S}{\partial x^{2}}-q(S) n,
\end{aligned}
$$

where $n(x, t)$ is the density of bacteria at time $t$ and position $x \in \mathbb{R}, S(x, t)$ is the concentration of the substrate (chemoattractant, chemotactic signal), $\chi(S)$ is the so-called chemotactic sensitivity (or chemotactic coefficient), $q(S)$ is the rate of consumption of substrate per cell, and $D_{n}$ and $D_{S}$ are the diffusion constants of bacteria and chemoattractant, respectively. While this theoretical framework capably generates chemotactic bands of motile bacteria, to develop formal stationary travelling bands (constant speed and shape) it is necessary to postulate a singular chemotactic sensitivity $\chi(S)$ in the limit $S \rightarrow 0$. More specifically, by 
applying the travelling wave ansatz $n(x, t)=\mathcal{N}(x-c t), S(x, t)=\mathcal{S}(x-c t)$ to $(1)$ where $\mathcal{N}(\xi), \mathcal{S}(\xi)$ are functions of a single variable $\xi$ and $c$ is the travelling speed of the wave, we obtain

$$
-c \mathcal{N}^{\prime}=D_{n} \mathcal{N}^{\prime \prime}-\left(\mathcal{N} \chi(\mathcal{S}) \mathcal{S}^{\prime}\right)^{\prime}
$$

where primes denote the derivatives. Integrating once and applying the natural boundary conditions $\mathcal{N}^{\prime}(\xi) \rightarrow$ $0, \mathcal{S}^{\prime}(\xi) \rightarrow 0, \mathcal{N}(\xi) \rightarrow 0$ as $\xi \rightarrow \infty$, we obtain

$$
-c \mathcal{N}=D_{n} \mathcal{N}^{\prime}-\mathcal{N} \chi(\mathcal{S}) \mathcal{S}^{\prime}
$$

Solving for $\mathcal{N}$, we get

$$
\mathcal{N}(\xi)=n_{0} \exp \left[-\frac{c}{D_{n}} \xi+\frac{1}{D_{n}} \int_{0}^{\xi} \chi(\mathcal{S}(\eta)) \mathcal{S}^{\prime}(\eta) \mathrm{d} \eta\right],
$$

where $n_{0}$ is a positive constant. Given $c>0$ and $\mathcal{S}(\eta) \searrow 0$ as $\eta \rightarrow-\infty$, we require $\chi(S) \rightarrow \infty$ as $S \rightarrow 0$ for $\mathcal{N}(\xi)$ to be bounded at $\xi=-\infty$, that is, $\chi(S)$ must be singular as $S \rightarrow 0$. For example, Keller and Segel $[23]$ used the form

$$
\chi(S)=\frac{k}{S}
$$

where $k$ is a constant. A number of further studies into travelling bands and waves in chemotaxis equations have been made $[18,19,26,27]$, yet a singularity of the form (3) is often necessary for the existence of travelling waves.

Singularities in the chemotactic sensitivity are problematic on multiple levels. Firstly, consider a singularity of the form (3). The macroscopic drift (velocity) satisfies

$$
\chi(S) \frac{\partial S}{\partial x}=k \frac{\partial}{\partial x}(\log (S)) .
$$

The above clearly exceeds biological ranges for cell speed: for $E$. coli these are typically in the range $10-$ $20 \mu \mathrm{m} / \mathrm{s}$. For example, if $S$ is an exponential signal ramp, $S \sim \exp (\lambda x)$, then the macroscopic drift is $k \lambda$, which exceeds $10-20 \mu \mathrm{m} / \mathrm{s}$ for suitable $\lambda$. Exponential signals were previously used in experiments [11,33], but the undesirable behaviour of macroscopic drift (as $S \rightarrow 0$ ) is not limited to them and can be observed for other signal functions.

Secondly, the limit $S \rightarrow 0$ corresponds to systems with very few copies of signalling molecules. Embedded in the PDE formulation (1)-(2) is an implicit assumption that the signal gradient $\partial S / \partial x$ is perfectly estimated by the cells, with high weighting $\chi(S)=k / S$ in the limit $S \rightarrow 0$. PDE modelling, however, is inapplicable in this limit due to the high levels of noise. Individual-based stochastic models under assumption (3) fail to generate travelling wave behaviour due to the intrinsic and external noise in the system preventing perfect sampling of the signal gradients. In this respect, the singularity (3) is not a robust mechanism for generating travelling bands in more detailed individual-based models.

One solution to the paradox of the singular chemotactic sensitivity lies in the argument that travelling waves and bands of motile bacteria observed in nature are not stationary but transient [8]. As such, a singularity in the chemotactic sensitivity may be unnecessary and, for example, a receptor binding based sensitivity [14,34] in (1)-(2), $\chi=a /(b+S)^{2}$ where $a$ and $b$ are constants, leads to decaying travelling bands that eventually die out. To generate travelling wave solutions in the strict mathematical sense, one can also introduce cell growth and death into the model. In [24], a logistic growth term is appended to equation (1), yet this approach will allow travelling wave solutions even in the absence of chemotaxis, as it is known, for example, for Fisher's equation $n_{t}=n_{x x}+n(1-n)$ [28]. Analytical and numerical evidence for traveling waves under multiple signal gradient in a different setup has also been given recently in [6].

In this paper we show that it is possible to obtain stationary travelling solutions without the unbounded velocity resulting from a singularity in the chemotactic sensitivity and without explicit growth terms or the introduction of additional attractant. We formulate a model for bacterial chemotaxis, consistent with the current biology, that employs transport equations for velocity-jump processes incorporating internal variables $[14,15,34]$ to describe signal transduction and metabolism. We provide the existence conditions for travelling wave solutions when coupled to the equations for external chemicals and study the form of these waves both numerically and analytically. The remainder of this paper is organized as follows. In Section 2, we introduce 

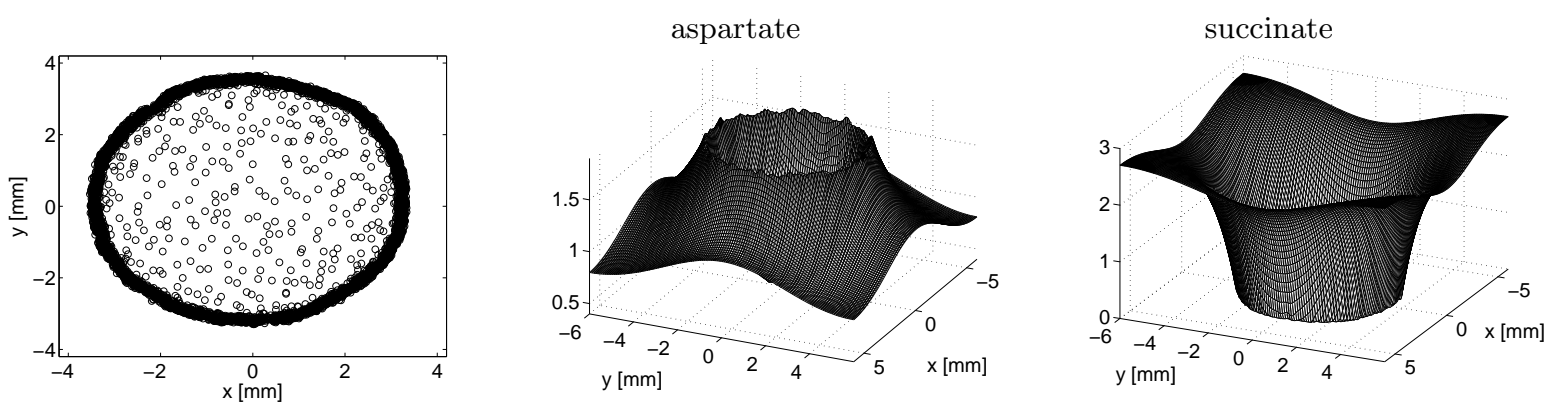

Fig. 1 Results of individual-based model presented in [12]. The propagating ring of cells (left), the concentration of aspartate (middle) and the concentration of succinate (right) at time 9 hours after inoculation. Reproduced from [12] with permission.

the hyperbolic chemotaxis model with internal variables describing signal transduction and cell metabolism. This model includes the same signal transduction and cell movement components as the hybrid cell-based model in [12], and a new approach for modeling the cell metabolism by introducing metabolic internal variables. We also demonstrate that the model is well-posed by proving the global existence of solutions. In Section 3, we report the results of a numerical investigation, indicating that the model may be capable of travelling wave solutions under certain limiting scenarios. This is formally proven in Section 4 . We conclude this paper with a brief discussion in Section 5 .

\section{A velocity jump model for bacterial chemotaxis incorporating internal variables}

The formulation of our model is based on the experimental situation reported in $[9,10]$, in which bacteria cells swim freely in a low agar concentration, consume the nutrient succinate and secrete the attractant aspartate. Different spatial patterns form when a small population of $E$. coli are inoculated into a chemotactically inert environment and stressed by various conditions, such as introduction of components of the TCA cycle (e.g. malate, fumarate or succinate), antibiotics, and cold shock. Under succinate application, its internalization provides the precursors for certain steps in the TCA cycle eventually resulting in the production and secretion of aspartate [15].

Initially, significant growth and division is observed at the site of inoculation. Bacteria consume succinate and secrete aspartate. As the aspartate concentration increases, they respond chemotactically and the density of the aggregate increases. This leads to local succinate depletion and a drop in aspartate production. Those cells near the aggregate boundary continue to receive succinate diffusing inwards and, hence, maintain aspartate production [15]. This creates a ring of aspartate-producing cells that moves radially outwards. Under low succinate concentrations the ring may propagate without breaking up $[9,10]$, the situation we focus on in this paper. In [12] a hybrid model was developed in which cells are treated as individual agents and external chemicals as continuous fields. A snapshot from the model is reproduced in Figure 1, showing the propagating ring of cells, a "volcano-shaped" profile of chemoattractant aspartate and the concentration of the primary nutrient source (succinate).

Here, we formulate a PDE model based on transport equations for a velocity-jump process. For simplicity we restrict to a one-dimensional geometry, representing either the radial spread of cells away from the inoculation site (in the context of the above experiments) or the movement of a band within a capillary tube. Since $E$. coli runs with a (more or less) constant speed $s=10-20 \mu \mathrm{m} / \mathrm{sec}$, we restrict their velocity to two values in the one-dimensional model, namely $v= \pm s$, where $s$ is the constant speed. The bacterial population is described by the two density functions, $p^{ \pm}(x, \mathbf{y}, \mathbf{z}, t)$, giving the density of cells at point $x$, velocity $v= \pm s$ and internal state $[\mathbf{y}, \mathbf{z}]$. The internal state here is stated with respect to two vectors: $\mathbf{y}$ describing signal transduction and $\mathbf{z}$ describing cellular metabolism. The coordinates of vector $\mathbf{y}$ represent the concentrations of various proteins in the cell and receptor states that are involved in the signal transduction. The coordinates of the metabolic vector $\mathbf{z}$ may include concentrations of the components in the TCA cycle and ATP. Detailed models of the signal transduction process have been developed [5,31] and are typically 
written as systems of ordinary differential equations

$$
\frac{\mathrm{d} \mathbf{y}}{\mathrm{d} t}=\mathbf{f}(\mathbf{y}, S),
$$

where $\mathbf{y} \in \mathbb{R}^{q}, \mathbf{f}=\left(f_{1}, f_{2}, \ldots, f_{q}\right): \mathbb{R}^{q+1} \rightarrow \mathbb{R}^{q}$ is a given right-hand side and $S(x, t)$ describes the concentrations of external signal at point $x$ and time $t$. These dynamical systems have bounded solutions of $\mathbf{y}$ for $t \in[0, \infty]$, given proper initial value, which corresponds to the fact that biological materials are conserved and do not blow up. The ensuing high-dimensionality of $\mathbf{y}$ hinders further mathematical and numerical analysis, however, it is possible to substitute the general model (4) with a low-dimensional system that captures essential features of the internal dynamics while still providing valuable biological insight. For example, a simplified two-dimensional toy model of the form (4) has been previously used to relate microscopic parameters of signal transduction and motor behaviour (i.e. excitation, adaptation times and turning frequency) with coefficients in the macroscopic partial differential equation (1) $[14,15,34,35]$. Similar questions for models of eukaryotic cells have also been addressed in $[16,35]$. In Section 3, we will assume fast signal transduction and simplify the model by eliminating the signal transduction variables, thus we do not specify the explicit form of $\mathbf{f}$ here. In this section, however, we state the general model using (4) and prove the global existence of its solutions, under the general assumption that (4) has bounded solutions in time for biologically realistic initial conditions, specifically, there exists a non-decreasing function $K(\cdot)$ such that

$$
|\mathbf{y}(t)| \leq K(\max |S|), \quad \text { for } \mathbf{y}(0) \leq K(\max |S|) .
$$

The dynamics of the metabolic internal variables $\mathbf{z}$ can, in general, be described by a system of ODEs in a similar vein to (4). The metabolic rates of the cell in turn depend on these internal variables. To reflect the experiments in $[9,10,8]$, where the signal $S(x, t)$ is aspartate and the food $F(x, t)$ is succinate, we propose here a cartoon description below with two variables $\mathbf{z}=\left(z_{1}, z_{2}\right)$, satisfying

$$
\frac{\mathrm{d} z_{i}}{\mathrm{~d} t}=g_{i}(\mathbf{z}, F), \quad i=1,2 .
$$

The metabolic processes to incorporate include: (i) bacteria consume succinate $F(x, t)$; (ii) cells secrete aspartate $S(x, t)$; and (iii) starving bacteria consume aspartate $S(x, t)$. To capture these processes, we assume an internal variable $z_{1}$ is produced from $F$ (succinate) which subsequently generates aspartate $S$ via the following straightforward metabolic pathway

$$
F \rightarrow z_{1} \rightarrow S .
$$

The variable $z_{1}$ may stand for fumarate in the TCA cycle, and low levels of $z_{1}$ correspond to a succinatestarved state in which case the cell may switch to consume aspartate instead [8]. We assume that $z_{1}$ catalytically influences production of a hypothetical "starving variable", $z_{2}$, according to

$$
\emptyset \stackrel{z_{1}}{\rightarrow} z_{2} \rightarrow \emptyset \text {. }
$$

Here, $\emptyset$ represents reactants/products assumed to be in excess. We assume mass action kinetics thus the functional forms for $g_{i}(\mathbf{z}, F)$ are linear in $z_{1}$ and $z_{2}$. We assume that

$$
g_{1}(\mathbf{z}, F)=\frac{F(x, t)-z_{1}}{t_{f}}, \quad g_{2}(\mathbf{z}, F)=\frac{z_{1}-z_{2}}{t_{m}},
$$

where $t_{f}$ is the characteristic time scale for the generation of the intermediate metabolic variable $z_{1}$, which can be seconds or fraction of seconds, and $z_{2}$ is the characteristic time scale for generation of the starving variable $z_{2}$, which is about 20 minutes [8].

Considering (4) and (6), the evolution of $p^{ \pm}(x, \mathbf{y}, \mathbf{z}, t)$ is described by the following equations

$$
\frac{\partial p^{+}}{\partial t}+s \frac{\partial p^{+}}{\partial x}+\sum_{i=1}^{q} \frac{\partial}{\partial y_{i}}\left[f_{i}(\mathbf{y}, S) p^{+}\right]+\sum_{i=1}^{2} \frac{\partial}{\partial z_{i}}\left[g_{i}(\mathbf{z}, F) p^{+}\right]=\lambda(\mathbf{y})\left[-p^{+}+p^{-}\right]+k(\mathbf{z}) p^{+},
$$




$$
\frac{\partial p^{-}}{\partial t}-s \frac{\partial p^{-}}{\partial x}+\sum_{i=1}^{q} \frac{\partial}{\partial y_{i}}\left[f_{i}(\mathbf{y}, S) p^{-}\right]+\sum_{i=1}^{2} \frac{\partial}{\partial z_{i}}\left[g_{i}(\mathbf{z}, F) p^{-}\right]=\lambda(\mathbf{y})\left[-p^{-}+p^{+}\right]+k(\mathbf{z}) p^{-} .
$$

In the above, the bacterial turning frequency $\lambda(\mathbf{y})$ depends on the signal transduction variables while the cell proliferation rate $k(\mathbf{z})$ depends on the metabolic variables.

We assume that for higher values of $z_{2}$ cells consume succinate and secrete aspartate, while for lower values of $z_{2}$ cells consume aspartate instead. Therefore, the equations for aspartate $(S)$ and succinate $(F)$ are

$$
\begin{aligned}
& \frac{\partial S}{\partial t}=D_{S} \frac{\partial^{2} S}{\partial x^{2}}+\alpha F \int_{\mathbb{Z}} \int_{\mathbb{Y}} h\left(z_{2}\right)\left[p^{+}(\mathbf{y}, \mathbf{z})+p^{-}(\mathbf{y}, \mathbf{z})\right] \mathrm{d} \mathbf{y} \mathrm{d} \mathbf{z} \\
& \quad-\beta S \int_{\mathbb{Z}} \int_{\mathbb{Y}}\left[1-h\left(z_{2}\right)\right]\left[p^{+}(\mathbf{y}, \mathbf{z})+p^{-}(\mathbf{y}, \mathbf{z})\right] \mathrm{d} \mathbf{y} \mathrm{d} \mathbf{z}-\gamma S, \\
& \frac{\partial F}{\partial t}=D_{F} \frac{\partial^{2} F}{\partial x^{2}}-\beta F \int_{\mathbb{Z}} \int_{\mathbb{Y}} h\left(z_{2}\right)\left[p^{+}(\mathbf{y}, \mathbf{z})+p^{-}(\mathbf{y}, \mathbf{z})\right] \mathrm{d} \mathbf{y} \mathrm{d} \mathbf{z} .
\end{aligned}
$$

where $\alpha, \beta>0, \gamma \geq 0$ and $h \equiv h\left(z_{2}\right):[0, \infty) \rightarrow[0,1]$ is an increasing function of $z_{2}$. Two explicit forms for the function $h$ will be considered: (i) a heaviside function representing a simple switch,

$$
h\left(z_{2}\right)=\left\{\begin{array}{l}
0 \text { for } z_{2} \leqslant z_{c} \\
1 \text { for } z_{2}>z_{c}
\end{array}\right.
$$

where the parameter $z_{c}$ represents a critical threshold for conversion to a starving phenotype; and (ii) a simple linear form $h\left(z_{2}\right)=a z_{2}$. We note that equation (10) describes both the production of the attractant (aspartate) $S$ by succinate-rich cells as well as its consumption by succinate-starved cells (when $h\left(z_{2}\right)$ is small) while equation (11) takes into account that succinate is the primary carbon source and will be consumed by cells when available. If $\gamma$ is positive, then equation (10) also includes the degradation of aspartate $(S)$ with the rate $\gamma$.

Motivated by a typical experimental set-up, we assume an initially uniform distribution of succinate and zero aspartate,

$$
F(x, 0) \equiv F_{0}>0, \quad S(x, 0) \equiv 0 .
$$

Cells are introduced at a single concentrated location and equally distributed into left- and right-moving populations (i.e. $p^{+}=p^{-}$at time $t=0$ ).

As discussed above, PDE models for chemotaxis with growth terms can have travelling wave solutions [24, 28], however the main goal of the present paper lies in determining whether "purely-chemotactic" travelling waves are possible in the absence of singular chemotactic sensitivities and unbounded velocities. We therefore simplify (8)-(9) by assuming

$$
k(\mathbf{z}) \equiv 0
$$

for the remainder of this paper. We note that the above imposes distinct biological considerations for different experimental set-ups. For the bands of bacteria observed when inoculated within the quasi one-dimensional geometry of a capillary tube, e.g. [2-4], the above simply assumes that no cell proliferation occurs. For the spreading rings seen when placed into the centre of a Petri dish it implicitly postulates that the stretch of the ring caused by wave movement is exactly compensated by the growth of cell number through proliferation.

Before we proceed to address the issue of travelling waves, we demonstrate the global existence of solutions to (8)-(11) with condition (14) under suitable growth assumptions:

Theorem 1 Let us assume (5), (7), (14) and that initial conditions satisfy

$$
p_{0}^{+} \in L^{1} \cap L^{\infty}\left(\mathbb{R} \times \mathbb{R}^{q} \times \mathbb{R}^{2}\right), \quad p_{0}^{-} \in L^{1} \cap L^{\infty}\left(\mathbb{R} \times \mathbb{R}^{q} \times \mathbb{R}^{2}\right), \quad F_{0} \in L^{\infty}(\mathbb{R}) \quad \text { and } \quad S_{0} \in L^{\infty}(\mathbb{R}),
$$

and

$$
p_{0}^{ \pm}(x, \mathbf{y}, \mathbf{z}) \equiv 0 \text { for }|\mathbf{y}| \geq K(0)
$$

Let us also assume that $\lambda(\mathbf{y})$ is bounded and piecewise linear and that there exist non-negative continuous functions $\Pi_{i}(\cdot) \in C(\mathbb{R}), i=1,2$, satisfying

$$
\left|\nabla_{\mathbf{y}} \cdot \mathbf{f}(\mathbf{y}, w)\right| \leq \Pi_{1}(|w|), \quad\left|\nabla_{\mathbf{z}} \cdot \mathbf{g}(\mathbf{z}, w)\right| \leq \Pi_{2}(|w|) .
$$


Then there exists a global solution of the system (8)-(11) satisfying, for all $t \geq 0$,

$$
\begin{gathered}
p^{+}(\cdot, \cdot, \cdot, t) \in L^{1} \cap L^{\infty}\left(\mathbb{R} \times \mathbb{R}^{q} \times \mathbb{R}^{2}\right), \quad p^{-}(\cdot, \cdot, \cdot, t) \in L^{1} \cap L^{\infty}\left(\mathbb{R} \times \mathbb{R}^{q} \times \mathbb{R}^{2}\right), \\
S(\cdot, t) \in L^{\infty}(\mathbb{R}), \quad F(\cdot, t) \in L^{\infty}(\mathbb{R}),
\end{gathered}
$$

and initial conditions $p^{+}(\cdot, \cdot, \cdot, 0)=p_{0}^{+}(\cdot, \cdot, \cdot), p^{-}(\cdot, \cdot, \cdot, 0)=p_{0}^{-}(\cdot, \cdot, \cdot), S(\cdot, 0)=S_{0}(\cdot)$ and $F(\cdot, 0)=F_{0}(\cdot)$.

An auxiliary lemma necessary for the proof and a sketch of the proof of the above theorem are given in Appendix A. It is worth noting that the assumption (15) includes the cartoon model for metabolism given by (7) and that we do not require continuity in $\lambda$. The elliptic case where $D_{S}=D_{F}=\infty$ can also be studied using the methods from $[7,13,22,20,21]$.

\section{Travelling waves - dependence on the turning kernel}

As discussed in Section 1, stationary travelling wave solutions are certainly possible in Keller-Segel type models (1)-(2) under either a singular chemotactic sensitivity or through the addition of an appropriate growth term. It is also anticipated that suitable $k(\mathbf{z})$ might lead to travelling solutions in (8)-(9), however here we remain focused on the zero growth case (14). To explore this, we employ a number of additional simplifying assumptions aimed at further improving the tractability of the model. As discussed in Section 2 , we have $t_{f} \ll t_{m}$. Hence we reduce equations (8)-(9) through the approximation $t_{f}=0$. Furthermore, considering that the adaptation time of signal transduction is of order seconds which is much smaller than $t_{m} \approx 20$ min, equations (8)-(9) can be further simplified by allowing cells to effectively sense the gradient of the external signal $S$ (i.e. no explicit representation for the internal signal transduction variables). Denoting $z=z_{2}$, the equations for $p^{+}(x, z, t)$ and $p^{-}(x, z, t)$ reduce to

$$
\begin{aligned}
& \frac{\partial p^{+}}{\partial t}+s \frac{\partial p^{+}}{\partial x}+\frac{\partial}{\partial z}\left(\frac{F-z}{t_{m}} p^{+}\right)=-\lambda\left(-\frac{\partial S}{\partial x}\right) p^{+}+\lambda\left(\frac{\partial S}{\partial x}\right) p^{-}, \\
& \frac{\partial p^{-}}{\partial t}-s \frac{\partial p^{-}}{\partial x}+\frac{\partial}{\partial z}\left(\frac{F-z}{t_{m}} p^{-}\right)=\lambda\left(-\frac{\partial S}{\partial x}\right) p^{+}-\lambda\left(\frac{\partial S}{\partial x}\right) p^{-} .
\end{aligned}
$$

Under the same simplifying assumptions (i.e. $z=z_{2}$ and no y), equations (10)-(11) are given by

$$
\begin{aligned}
& \frac{\partial S}{\partial t}=D_{S} \frac{\partial^{2} S}{\partial x^{2}}+\alpha F \int_{\mathbb{R}} h(z)\left[p^{+}(z)+p^{-}(z)\right] \mathrm{d} z-\beta S \int_{\mathbb{R}}[1-h(z)]\left[p^{+}(z)+p^{-}(z)\right] \mathrm{d} z-\gamma S, \\
& \frac{\partial F}{\partial t}=D_{F} \frac{\partial^{2} F}{\partial x^{2}}-\beta F \int_{\mathbb{R}} h(z)\left[p^{+}(z)+p^{-}(z)\right] \mathrm{d} z .
\end{aligned}
$$

The system of equations (18)-(21) is a simplification of a more general system (8)-(11) for which the existence of solutions was established in Theorem 1. However, since $\lambda$ is assumed to directly depend on the signal gradient, the system (18)-(21) is not written in the form (8)-(11). Theorem 1 cannot be directly applied to establish that the system (18)-(21) is also well-posed. Consequently, we formulate the existence of solutions of the system (18)-(21) as the following theorem.

Theorem 2 Let initial conditions satisfy

$$
p_{0}^{+} \in L^{1} \cap L^{\infty}(\mathbb{R} \times \mathbb{R}), \quad p_{0}^{-} \in L^{1} \cap L^{\infty}(\mathbb{R} \times \mathbb{R}), \quad F_{0} \in W^{2, r}(\mathbb{R}) \quad \text { and } \quad S_{0} \in W^{2, r}(\mathbb{R}),
$$

for all $1 \leq r<\infty$. Let us assume that

$$
\lambda(\zeta) \leq C(1+|\zeta|) .
$$

Then there exists a global solution of the system (18)-(21) satisfying, for all $t \geq 0$

$$
\begin{gathered}
p^{+}(\cdot, \cdot, t) \in L^{1} \cap L^{\infty}(\mathbb{R} \times \mathbb{R}), \quad p^{-}(\cdot, \cdot, t) \in L^{1} \cap L^{\infty}(\mathbb{R} \times \mathbb{R}), \\
S(\cdot, t) \in W^{2, r}(\mathbb{R}), \quad F(\cdot, t) \in W^{2, r}(\mathbb{R}), \quad \text { for all } 1 \leq r<\infty,
\end{gathered}
$$

and initial conditions $p^{+}(\cdot, \cdot, 0)=p_{0}^{+}(\cdot, \cdot), p^{-}(\cdot, \cdot, 0)=p_{0}^{-}(\cdot, \cdot), S(\cdot, 0)=S_{0}(\cdot)$ and $F(\cdot, 0)=F_{0}(\cdot)$. 
The proof of this theorem is similar to the proof of Theorem 1. It is sketched in Appendix A.

For the remainder of this section, we specify the turning rate as $\lambda: \mathbb{R} \rightarrow[0, \infty)$ :

$$
\lambda(\zeta)=\lambda_{0}\left(1+\frac{\zeta}{\kappa+|\zeta|}\right)
$$

where $\lambda_{0}$ is defined as the unbiased turning rate and $\kappa$ is a sensitivity coefficient. We note that under the limit $\kappa \rightarrow \infty, \lambda(\zeta)$ simply becomes the constant turning rate function $\lambda(\zeta)=\lambda_{0}$. Conversely, under the limit $\kappa \rightarrow 0$, we obtain the switch turning rate:

$$
\lambda(\zeta)= \begin{cases}0, & \text { if } \zeta<0 \\ \lambda_{0}, & \text { if } \zeta=0 \\ 2 \lambda_{0}, & \text { if } \zeta>0\end{cases}
$$

Both (25) and (26) satisfy the growth estimate (22), i.e Theorem 2 implies the global existence of solutions to the system $(18)-(21)$.

During the remainder of this section we present numerical solutions of (18)-(21) under the turning rate (25). For the numerical simulations we consider a one-dimensional domain $[-L, L]$ with zero-flux boundary conditions. Under the a priori assumption that the uniform initial succinate concentration has been scaled to unity, we set

$$
F(x, 0)=1, \quad S(x, 0)=0 .
$$

From (21) we note that for biologically relevant cases, $F$ is bounded between 0 and 1 and the crucial range for our internal variable is therefore $z \in[0,1]$. We choose $h(z)=z$ and, once again taking an a priori scaling assumption, we set

$$
p^{+}(x, z, 0)=p^{-}(x, z, 0)=e^{-a x^{2}} e^{-b(1-z)^{2}},
$$

Under suitably large values of $a$ and $b$, the above imposes an initial population of cells dropped into the centre of the domain and of a nonstarving phenotype (i.e. $h(z) \sim 1$ ).

Motivated by typical experimental conditions, we consider a one-dimensional domain of length $8 \mathrm{~cm}$ $(L=4)$ and zero-flux boundary conditions. We set $a=1000, b=2000$ while fixing the majority of parameters at the following set of biologically relevant values: $s=10 \mu \mathrm{m} \mathrm{s}^{-1}, \lambda_{0}=1 \mathrm{~s}^{-1}, t_{m}=30 \mathrm{~min}$ and $D_{S}=D_{F}(=$ $D)=10^{-5} \mathrm{~cm}^{2} \mathrm{~s}^{-1}$. We note that under the a priori scaling assumptions above, parameters $\alpha, \beta$ and $\gamma$ all have dimension $1 / T$ and we set $\alpha=\beta=1000 /$ hour with $\gamma=0$. Note that the high values for $\alpha$ and $\beta$ model the scenario in which succinate becomes rapidly depleted at the initial drop site.

To solve the equations we adopt a Method of Lines approach. Space $(x \in[-4,4])$ and the internal variable $(z \in[0,1])$ are both discretised using uniform meshes of discretisation lengths $\Delta x=0.004 \mathrm{~cm}$ and $\Delta z=0.01$, respectively. Cellular movement terms are discretised with third order upwinding, augmented by a Van Leer flux limiting scheme to maintain positivity of solutions. The internal variable terms in the cell equation are discretised with a first order upwinding scheme. The integral terms in the succinate/aspartate equations (20)(21) are approximated by a simple trapezoidal scheme while diffusion terms are solved via a second order central difference scheme. The resultant system of ODEs is integrated in time using the ROWMAP stiff system solver [32] using absolute and relative error tolerances fixed at $10^{-9}$. We note that similar methods have been successfully adopted to solve a number of related equations (e.g. see [17,29]). Validation of the numerical scheme has been performed through varying both discretisation lengths and error tolerances.

\subsection{Constant turning rates}

We first consider the case with a constant turning rate $\lambda(\zeta)=\lambda_{0}$. This corresponds to $\kappa \rightarrow \infty$ in $(25)$. Here, there is no bias in the response of cells to the aspartate gradient and we expect the bacteria to simply disperse from the initial drop location. In the left column of Figure 2 we plot the spatio-temporal evolution for the model variables, while in the right column we plot the cross-sectional profiles of the same variables at the specific time points $t=1$ hour (dotted line), $t=12$ hours (dash-dot line), $t=24$ hours (dashed line), $t=36$ hours (solid line) and $t=48$ hours (thick solid line). From top to bottom, the variables plotted represent the sum of right- and left-moving cells $\left(p^{+}+p^{-}\right)$integrated over the internal variable (i.e. the 

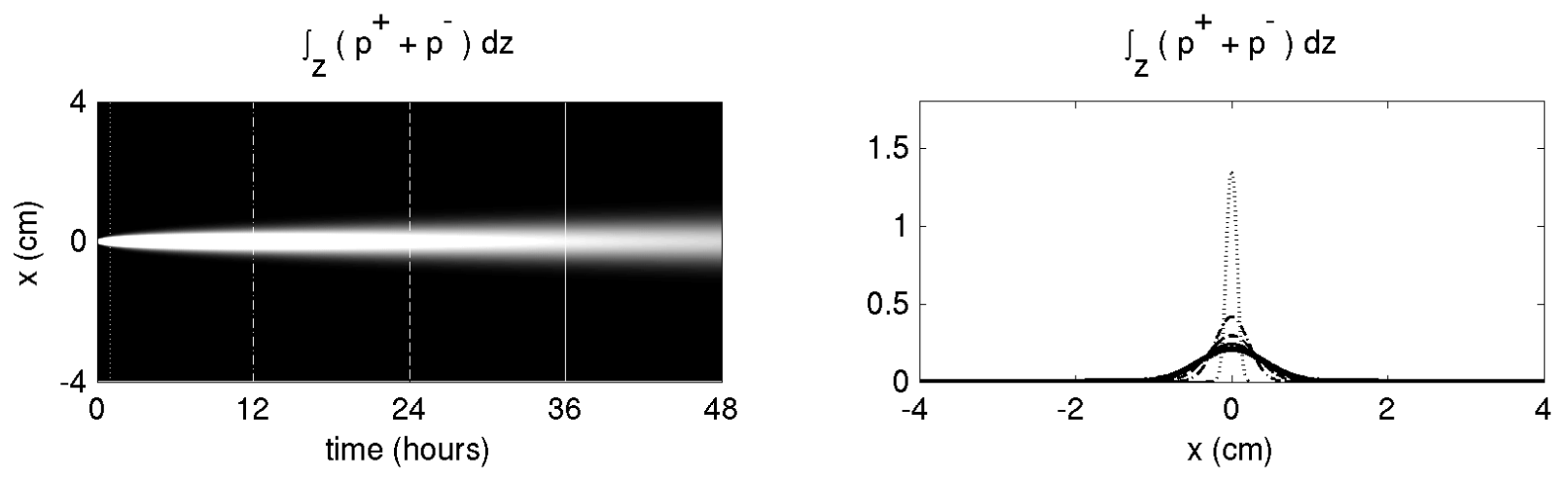

$$
\int_{x}\left(p^{+}+p^{-}\right) d x
$$

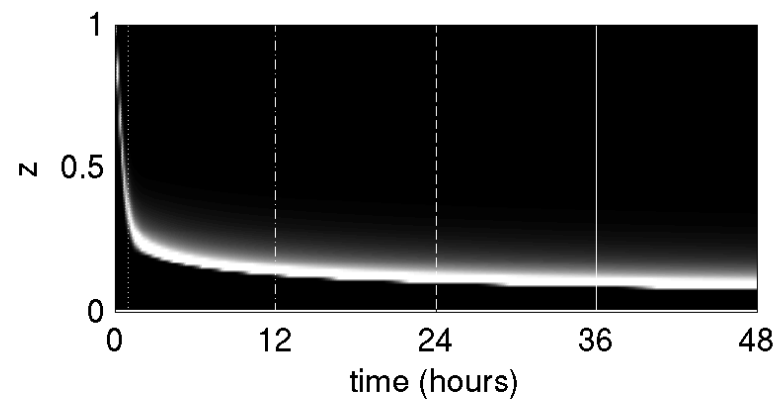

$\int_{x}\left(p^{+}+p^{-}\right) d x$

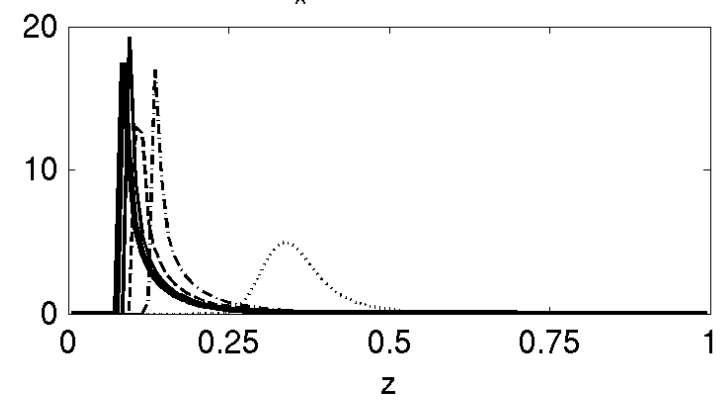

S (aspartate)
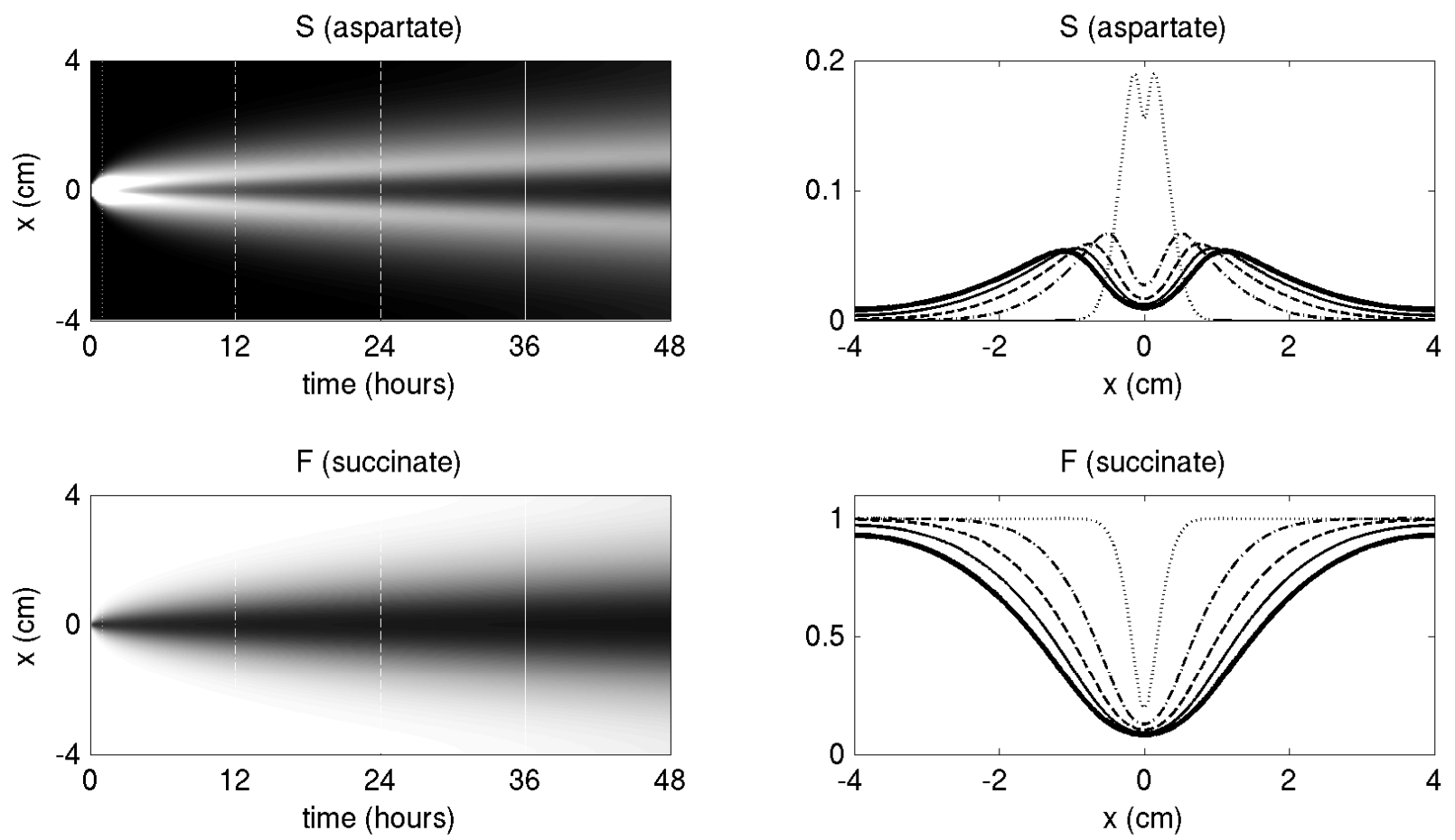

Fig. 2 Numerical simulations showing evolution of (18)-(21) under a constant turning rate $\left(\lambda_{0}\right)$. Left column show the spatio-temporal evolution of key variables, with brightness representing the magnitude of the variables. Right column plots the profiles at times $t=1$ (dotted line), 12 (dash-dot), 24 (dashed), 36 (solid) and 48 (thick solid) hours. Top row: macroscopic cell density, Second row: distribution of cells with respect to the internal variable $z$, Third row: aspartate concentration, Bottom row: succinate concentration. Model parameters and numerical details as described in text. 
macroscopic cell density $\left.n=\int\left(p^{+}+p^{-}\right) d z\right)$, the sum of right- and left-moving cells $\left(p^{+}+p^{-}\right)$integrated over space (i.e. the distribution of cells across the internal variable), the aspartate concentration and the succinate concentration.

Cells quickly ( $t=1$ hour profiles) deplete the available succinate (Fig. 2, bottom row) while producing aspartate (third row). Within areas of depleted succinate, cell starvation sets in accompanied by the subsequent metabolic shift to aspartate consumption (implied by the shift in the distribution of the cells with respect to the internal variable, second row). By $t=12$ hours, the aspartate is also depleted at the initial drop location. With zero chemotactic bias, cells undergo an unbiased random walk, resulting in the diffusive spread of cells from the initial drop location (Fig. 2, top row).

\subsection{Aspartate chemotaxis: Nonconstant turning rates}

We now incorporate chemotactic responses to aspartate gradients via the nonlinear turning rate (25) under varying $\kappa$. We note that smaller $\kappa$ leads to more significant responses under shallow signal gradients. Note that in the limit $\kappa \rightarrow 0$ we obtain the switch turning rate function (26), corresponding to infinite sensitivity to the signal (which is represented by the rate of change of $\lambda$ at 0 , i.e., $\lambda^{\prime}(0)$ for $\lambda$ differentiable).

In Fig. 3 we plot numerical simulations for (18)-(21) with (25) under the previously defined parameter values and $\kappa=1.0$. The addition of aspartate chemotaxis dramatically alters solution behaviour. In the initial phase $(t=1$ hour), the population remains concentrated near the initial drop location: rapid succinate depletion leads to aspartate production and chemotactic-induced aggregation of the population. The low succinate levels also induce a shift to a starving phenotype and subsequent aspartate consumption. Correspondingly, aspartate also becomes depleted at the inoculation site with aspartate maxima moving outwards from the inoculation site. The chemotactic response allows cells to keep pace with the aspartate maxima, generating bands of bacteria that migrate outwards. Note, however, that a certain fraction of the population are left within the tail of the band (where the aspartate gradient is low) - compare Fig. 4 (a) with (b) for an expanded view of the tail- resulting in the gradual reduction of the peak macroscopic density. This is clearly consistent with the snapshot of the hybrid based model of [12] shown in Figure 1. Nevertheless, this decrease remains relatively small on experimentally relevant timescales.

We further investigate the dependence of the band profile on the sensitivity of cells to the chemoattractant. In Fig. 4 we plot the bacterial bands under a range of $\kappa$ values. Larger values (e.g. Fig. 4 (c), $\kappa=10.0$ ) result in rapidly dispersing bands with the peak density dropping rapidly as it moves radially outwards. Reductions in $\kappa$ (e.g. Fig. 4 (d), $\kappa=0.1$ ) result in a more tightly maintained band that migrates with almost constant speed. Of further remark is the noticeably slower movement of the band under lower values of $\kappa$.

From a biological perspective, these results are clearly consistent with the experimental system described earlier, validating our modelling process. In the next section we show analytically that the migrating bands observed numerically here do not stabilize into travelling bands and eventually damp out, however in the limit $\kappa \rightarrow 0$ the migrating band does stabilize.

\section{Travelling wave analysis}

In this section, we analyse the existence of travelling wave solutions of the system (18)-(21) under different forms of turning rate $\lambda$ and compare the analytical results with the numerical solutions presented in Section 3. We assume $\lambda(0)=\lambda_{0}>0$ throughout the section, as suggested by biological observations. For analytical convenience, we define the $z$-moments,

$$
\begin{aligned}
n^{+}(x, t)=\int_{\mathbb{R}} p^{+}(x, z, t) \mathrm{d} z, & n^{-}(x, t)=\int_{\mathbb{R}} p^{-}(x, z, t) \mathrm{d} z, \\
n_{z}^{+}(x, t)=\int_{\mathbb{R}} z p^{+}(x, z, t) \mathrm{d} z, & n_{z}^{-}(x, t)=\int_{\mathbb{R}} z p^{-}(x, z, t) \mathrm{d} z,
\end{aligned}
$$

so the macroscopic cell density $n$ satisfies $n=n^{+}+n^{-}$. We further define,

$$
j=s\left(n^{+}-n^{-}\right), \quad n_{z}=n_{z}^{+}+n_{z}^{-}, \quad j_{z}=s\left(n_{z}^{+}-n_{z}^{-}\right) .
$$



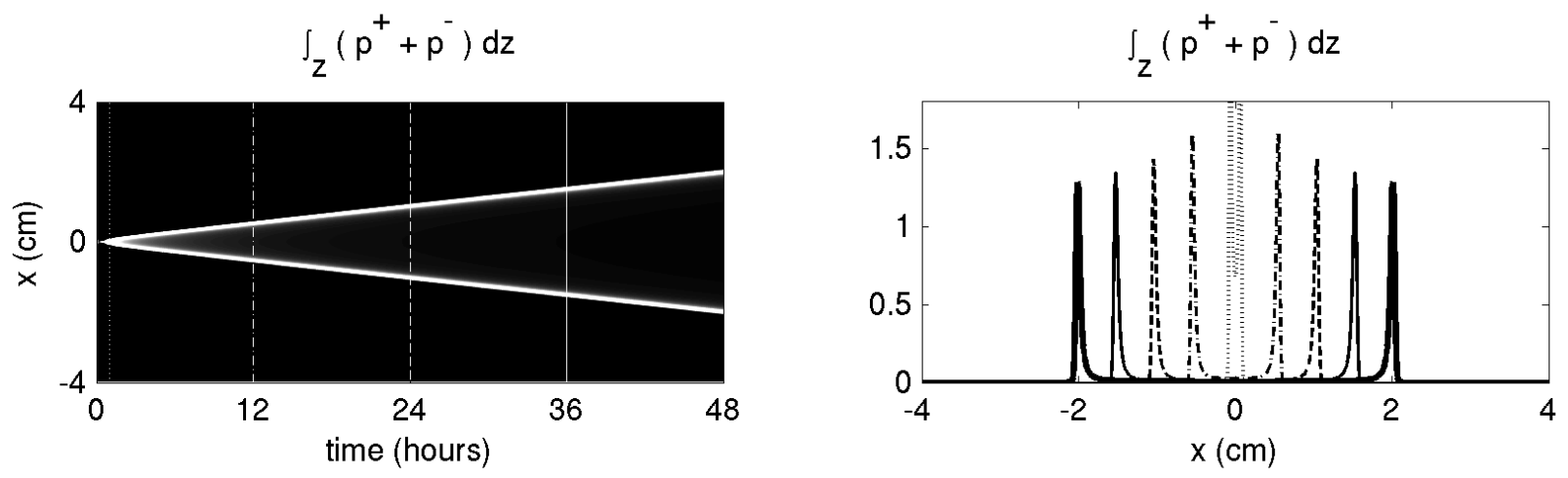

$$
\int_{x}\left(p^{+}+p^{-}\right) d x
$$

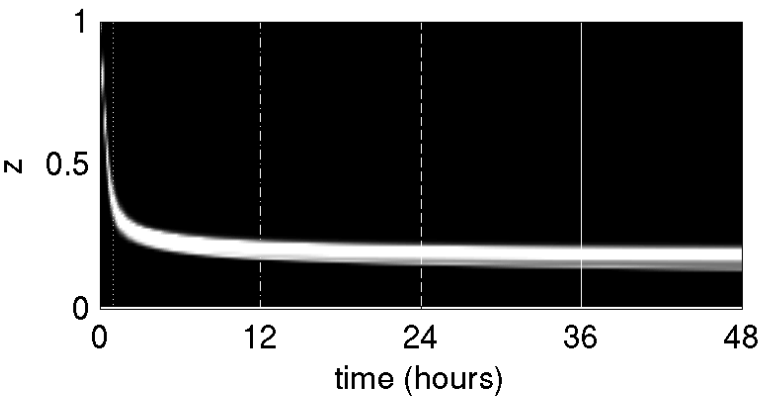

$$
\int_{x}\left(p^{+}+p^{-}\right) d x
$$

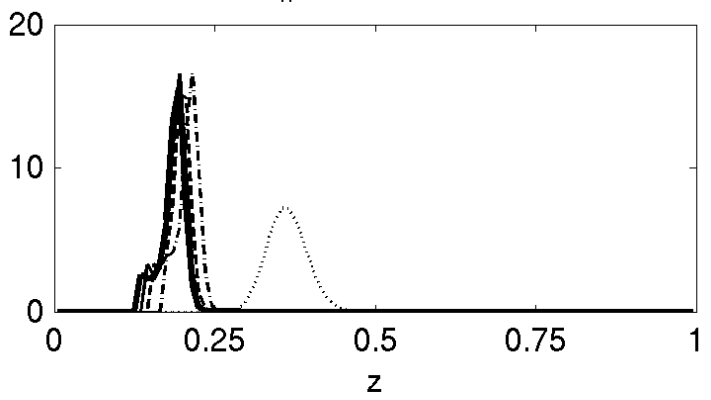

$S$ (aspartate)

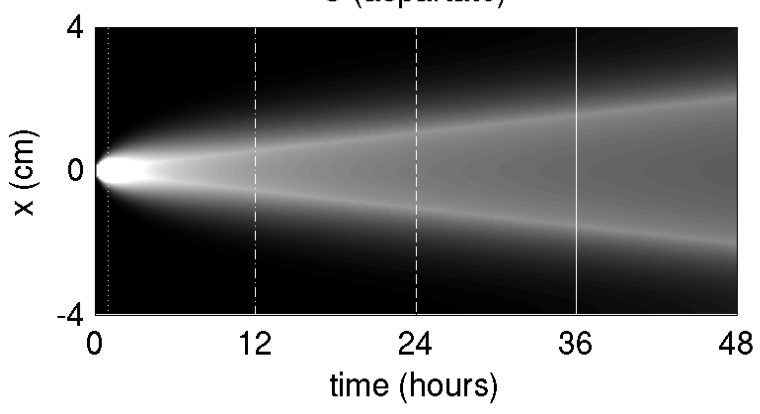

$\mathrm{S}$ (aspartate)

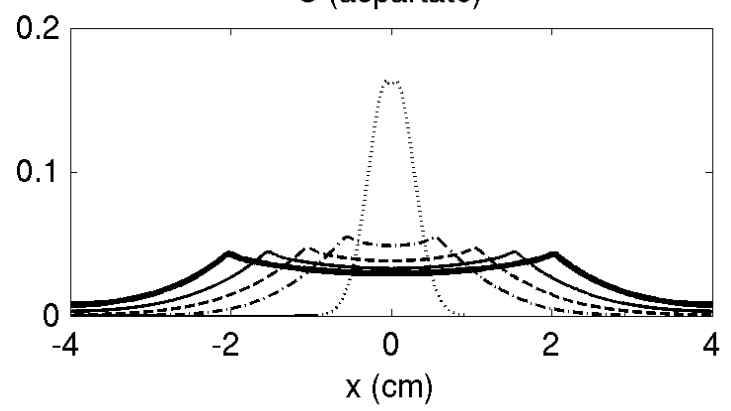

F (succinate)

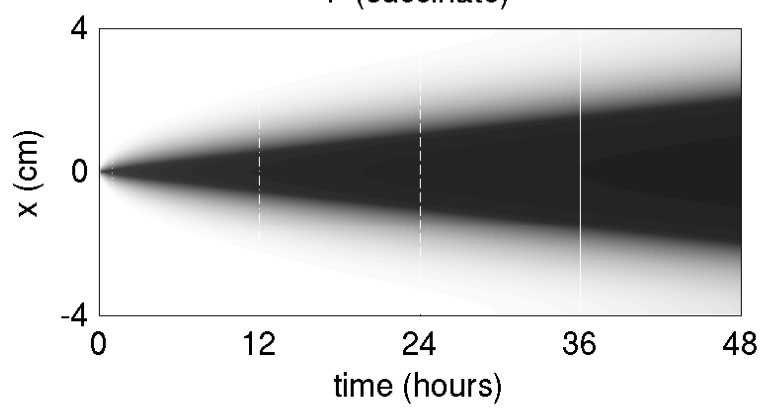

F (succinate)

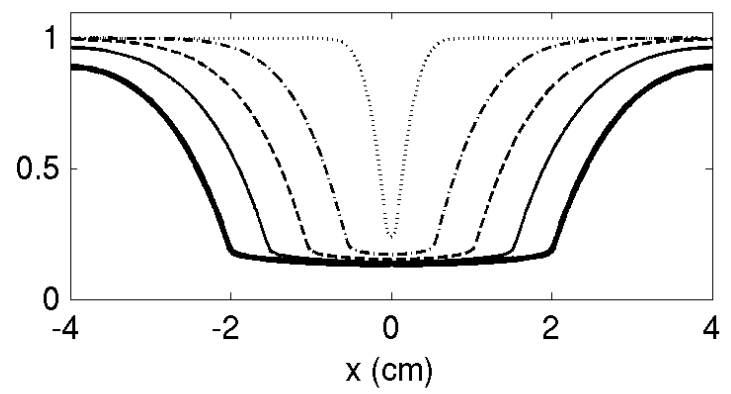

Fig. 3 Numerical simulations showing evolution of system (18)-(21) and (25) with $\kappa=1.0$. Left column show the spatio-temporal evolution of key variables while right column plots the profiles at times $t=1$ (dotted line), 12 (dashdot), 24 (dashed), 36 (solid) and 48 (thick solid) hours. Top row: macroscopic cell density, Second row: distribution of cells with respect to the internal variable $z$, Third row: aspartate concentration, Bottom row: succinate concentration. Model parameters and numerical details as described in text. 


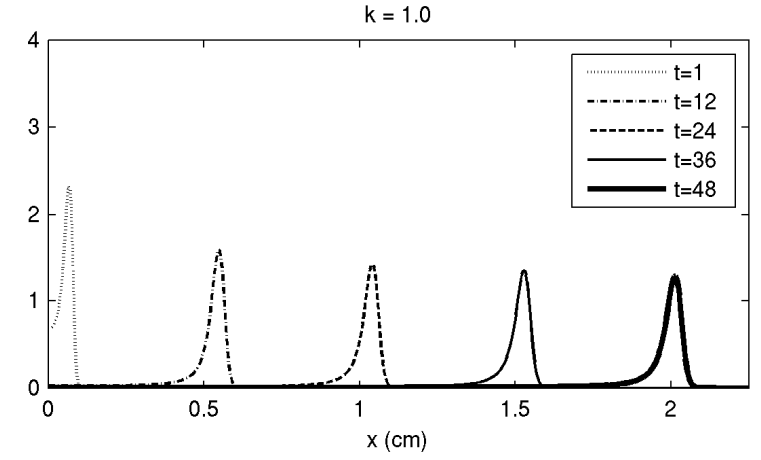

(a)

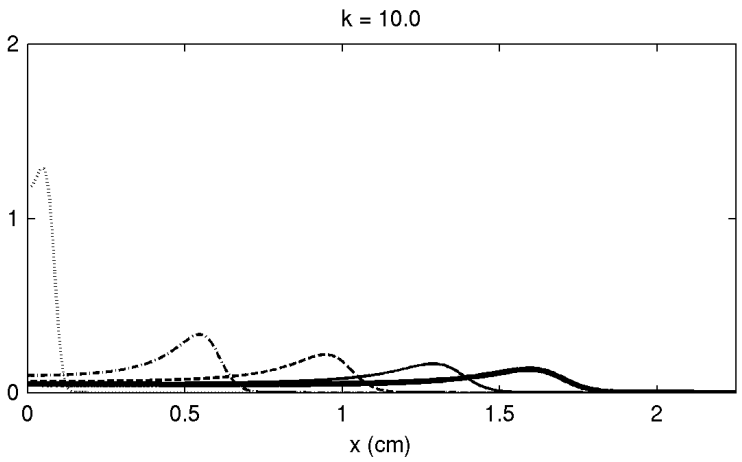

(c)

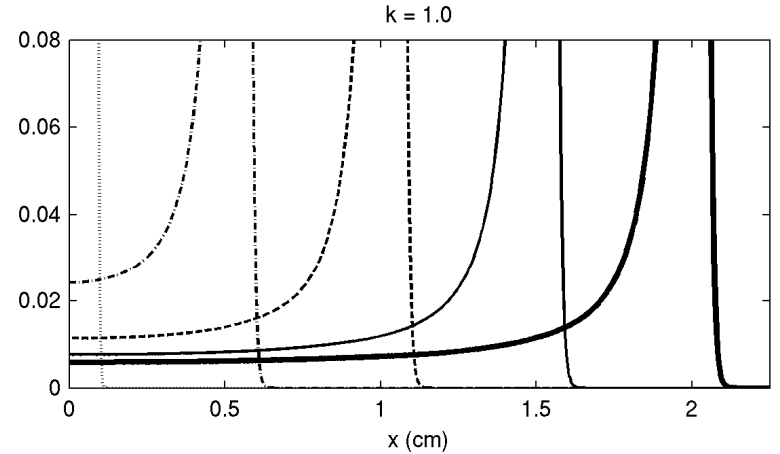

(b)

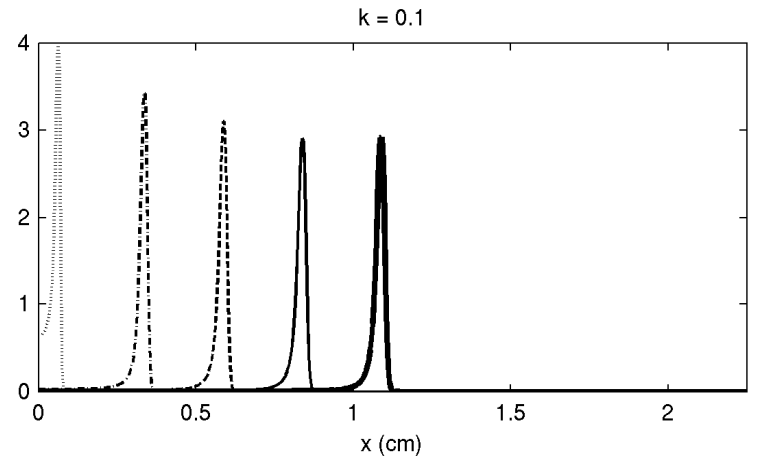

(d)

Fig. 4 Numerical simulations of (18)-(21) and (25) showing evolution of the macroscopic cell density under varying $\kappa$. (a) $\kappa=1$, (b) Expanded vertical axis view of $\kappa=1$ showing fraction of population left in the wake of the migrating band, (c) $\kappa=10,(d) \kappa=0.1$. For clarity, the solutions are plotted on the half domain $x \geq 0$. For each figure we plot solution profiles at $t=1$ (dotted), 12 (dash-dot), 24 (dashed), 36 (solid) and 48 (thick solid) hours. Other model parameters and numerical details as described in text.

We denote

$$
\lambda_{1}(\zeta) \equiv \lambda(\zeta)-\lambda(-\zeta) \quad \text { and } \quad \lambda_{2}(\zeta)=\lambda(\zeta)+\lambda(-\zeta)
$$

Then by integrating (18) - (19) over $z$, and adding (resp. subtracting and multiplying by $s$ ) the resulting equations, we obtain

$$
\begin{aligned}
& \frac{\partial n}{\partial t}+\frac{\partial j}{\partial x}=0 \\
& \frac{\partial j}{\partial t}+s^{2} \frac{\partial n}{\partial x}=s \lambda_{1}\left(\frac{\partial S}{\partial x}\right) n-\lambda_{2}\left(\frac{\partial S}{\partial x}\right) j .
\end{aligned}
$$

Multiplying (18) - (19) by $z$, integrating over $z$, and adding (resp. subtracting) the resulting equations, we get

$$
\begin{aligned}
& \frac{\partial n_{z}}{\partial t}+\frac{\partial j_{z}}{\partial x}=\frac{F}{t_{m}} n-\frac{1}{t_{m}} n_{z} \\
& \frac{\partial j_{z}}{\partial t}+s^{2} \frac{\partial n_{z}}{\partial x}=\frac{F}{t_{m}} j-\frac{1}{t_{m}} j_{z}+s \lambda_{1}\left(\frac{\partial S}{\partial x}\right) n_{z}-\lambda_{2}\left(\frac{\partial S}{\partial x}\right) j_{z}
\end{aligned}
$$

Equations (28), (29) are equivalent to

$$
\frac{\partial^{2} n}{\partial t^{2}}-s^{2} \frac{\partial^{2} n}{\partial x^{2}}=-s \frac{\partial}{\partial x}\left[\lambda_{1}\left(\frac{\partial S}{\partial x}\right) n\right]+\frac{\partial}{\partial x}\left[\lambda_{2}\left(\frac{\partial S}{\partial x}\right) j\right],
$$


from which we see that the hyperbolic chemotaxis model can only be reduced to classical chemotaxis equations when the turning rate is symmetric about $\lambda(0)=\lambda_{0}$ (i.e. when $\lambda(\zeta)-\lambda_{0}$ is an odd function of $\zeta$ ). To simplify equations (20), (21), we define the average value of $h$ at each point $x$ and time $t$ by

$$
H(x, t)=\int_{\mathbb{R}} h(z)\left[p^{+}(x, z, t)+p^{-}(x, z, t)\right] \mathrm{d} z,
$$

which represents the macroscopic density of the non-starving population if we regard $h(z)$ as the probability to be non-starving for cells with state variable $z$. Equations (20), (21) subsequently read as

$$
\begin{aligned}
& \frac{\partial S}{\partial t}=D_{S} \frac{\partial^{2} S}{\partial x^{2}}+\alpha F H-\beta S(n-H)-\gamma S, \\
& \frac{\partial F}{\partial t}=D_{F} \frac{\partial^{2} F}{\partial x^{2}}-\beta F H .
\end{aligned}
$$

In the remaining of this section, we first introduce the traveling wave ansatz, and then prove that as a necessary condition for traveling wave solutions, the turning rate $\lambda$ must be discontinuous at $\xi=0$, and this necessary condition is independent of the chemical dynamics, i.e., the equations for $S$ and $F$. This strictly proves that for $\kappa \neq 0$ in (25), the numerical waves will not stabilize to constant magnitude and speed. We finally study the existence of traveling wave solutions with the limiting turning rate (26) for two types of traveling waves:

Case I: no-starvation traveling waves. In this scenario, none of the cells starve, i.e. $p^{ \pm}(x, z, t) \equiv 0$ for $z \leq z_{c}$ when $h(z)$ is given by (12). Therefore $H \equiv n$ and $F \geq z_{c}$ globally. The system (28)-(29), (32)-(33) subsequently becomes a closed system of four equations for four unknowns $n, j, S$ and $F$.

Case II: traveling waves with starvation. In this case, equations (30)-(31) cannot be decoupled from the system (28) - (33) as for Case I. We note that the analysis conducted corresponds to the numerical exploration in Section 3 where we assumed $0 \leq \min F \leq \max F \leq 1$ and adopted the simple linear form $h(z)=z$. Hence we have $H=n_{z}$ and (28) - (33) is a closed system of six equations for six unknowns $n$, $j, n_{z}, j_{z}, S$ and $F$.

For the simplicity of the analysis we assume $D_{S}=D_{F}=0$ here. While we believe similar results are true for $D_{S}>0, D_{F}>0$, this remains the subject for future investigations.

\subsection{Travelling wave ansatz}

We take the travelling wave ansatz (with $0<c \leq s$ ),

$$
\begin{aligned}
n(x, t) & =\mathcal{N}(x-c t), \quad j(x, t)=\mathcal{J}(x-c t), \quad S(x, t)=\mathcal{S}(x-c t), \quad H(x, t)=\mathcal{H}(x-c t), \\
n_{z}(x, t) & =\mathcal{N}_{z}(x-c t), \quad j_{z}(x, t)=\mathcal{J}_{z}(x-c t), \quad F(x, t)=\mathcal{F}(x-c t),
\end{aligned}
$$

and substitute into equations (28)-(33). From (28) we obtain

$$
-c \mathcal{N}^{\prime}+\mathcal{J}^{\prime}=0
$$

which describes a conservation condition for the total cell mass. Assuming $\mathcal{J}$ and $\mathcal{N}$ decay to zero at infinity, the above implies

$$
\mathcal{J}=c \mathcal{N}
$$

If $c=s$ then, from equation (34) and the definitions of $n$ and $j$ we obtain $n^{-}=0$ and $n=n^{+}$: all cells swim to the right and tumbling does not occur irrespective of the signal. Therefore $\mathcal{N}(x-s t)=n(x, t)=$ $n^{+}(x, t)=n(x-s t, 0)$ and $\mathcal{N}_{z}(x-s t)=n_{z}(x, t)=n_{z}^{+}(x, t)=n_{z}(x-s t, 0)$. This case is trivial and we neglect 
it from further consideration. For the remainder of the paper, we look for travelling wave solutions in which $0<c<s$. Applying the traveling wave ansatz to (29)-(33) and using (34) we obtain

$$
\begin{aligned}
& \mathcal{N}^{\prime}=\frac{1}{s^{2}-c^{2}}\left(s \lambda_{1}\left(\mathcal{S}^{\prime}\right)-c \lambda_{2}\left(\mathcal{S}^{\prime}\right)\right) \mathcal{N} \\
& \mathcal{N}_{z}^{\prime}=\frac{1}{s^{2}-c^{2}}\left[\frac{2 c}{t_{m}} \mathcal{F} \mathcal{N}-\frac{c}{t_{m}} \mathcal{N}_{z}-\frac{1}{t_{m}} \mathcal{J}_{z}+s \lambda_{1}\left(\mathcal{S}^{\prime}\right) \mathcal{N}_{z}-\lambda_{2}\left(\mathcal{S}^{\prime}\right) \mathcal{J}_{z}\right] \\
& \mathcal{J}_{z}^{\prime}=\frac{1}{s^{2}-c^{2}}\left[\frac{s^{2}+c^{2}}{t_{m}} \mathcal{F} \mathcal{N}-\frac{s^{2}}{t_{m}} \mathcal{N}_{z}-\frac{c}{t_{m}} \mathcal{J}_{z}+c s \lambda_{1}\left(\mathcal{S}^{\prime}\right) \mathcal{N}_{z}-c \lambda_{2}\left(\mathcal{S}^{\prime}\right) \mathcal{J}_{z}\right]
\end{aligned}
$$

and

$$
\begin{aligned}
& -c \mathcal{S}^{\prime}=D_{S} \mathcal{S}^{\prime \prime}+\alpha \mathcal{F H}-\beta \mathcal{S}(\mathcal{N}-\mathcal{H})-\gamma \mathcal{S}, \\
& -c \mathcal{F}^{\prime}=D_{F} \mathcal{F}^{\prime \prime}-\beta \mathcal{F} \mathcal{H} .
\end{aligned}
$$

Steady states of the system satisfy $\left(\mathcal{N}, \mathcal{N}_{z}, \mathcal{J}_{z}, \mathcal{S}, \mathcal{F}\right)=\left(0,0,0, \mathcal{S}_{s}, \mathcal{F}_{s}\right)$. Travelling wave solutions correspond to heteroclinic orbits between different steady states, i.e. solutions of the system that satisfy

$$
\begin{aligned}
\lim _{\xi \rightarrow \pm \infty} \mathcal{N}(\xi) & =\lim _{\xi \rightarrow \pm \infty} \mathcal{N}_{z}(\xi)=\lim _{\xi \rightarrow \pm \infty} \mathcal{J}_{z}(\xi)=0, \\
\lim _{\xi \rightarrow \pm \infty} \mathcal{F}(\xi) & =F_{ \pm} \\
\lim _{\xi \rightarrow \pm \infty} \mathcal{S}(\xi) & =\mathcal{S}_{ \pm} \text {with } \mathcal{S}_{+}=0 .
\end{aligned}
$$

\subsection{A necessary condition for travelling wave solutions}

In Section 3 travelling wave-like solutions were numerically observed under the nonlinear function (25). For $\kappa>0$, these solutions decayed slowly with the decay rate decreasing as $\kappa \rightarrow 0$. Our speculation was that in the limit $\kappa \rightarrow 0$ travelling wave solutions may exist. In the following lemma we show from equation (35) that the existence of travelling wave solutions necessitates $\lambda$ to be discontinuous at 0 . Thus, for the turning rate (25) with $\kappa>0$, steady travelling waves in the strict mathematical sense do not exist.

Lemma 1 A nontrivial (i.e. $c \neq 0, \pm s)$ continuous travelling wave solution of (28)-(33) is only permissible when $\lambda(\zeta)$ is discontinuous at $\zeta=0$.

Proof. Denoting $V=\mathcal{S}^{\prime}$, equation (35) reads

$$
\mathcal{N}^{\prime}=\frac{1}{s^{2}-c^{2}}\left(s \lambda_{1}(V)-c \lambda_{2}(V)\right) \mathcal{N}
$$

We assume $\lambda(\zeta)$ is continuous at $\zeta=0$ and derive a contradiction. First assume that $\mathcal{N}>0$ in the whole domain. By the boundary conditions, $\mathcal{N} \rightarrow 0$ as $\xi \rightarrow-\infty$, therefore we have $\mathcal{N}^{\prime}\left(\xi_{j}\right)>0$ for $\xi_{j}<0$ and $\lim _{j \rightarrow \infty} \xi_{j}=-\infty$, which means $s \lambda_{1}\left(V\left(\xi_{j}\right)\right)-c \lambda_{2}\left(V\left(\xi_{j}\right)\right)>0$. However, since $V \rightarrow 0$ as $\xi \rightarrow-\infty$, by the continuity of $\lambda, s \lambda_{1}(V)-c \lambda_{2}(V) \rightarrow-2 c \lambda(0)<0$ as $\xi \rightarrow-\infty$ which gives a contradiction. Hence there is a finite negative number $\xi_{0}$ such that $n=0$ when $\xi \leq \xi_{0}$, and $n$ is not always equal to 0 for $\xi>\xi_{0}$. However, this is inconsistent with the uniqueness of the solution to equation (41), giving a contradiction. Therefore $\lambda(\zeta)$ can not be continuous at $\zeta=0$.

In the limiting case $\kappa \rightarrow 0$, the nonlinear turning rate (25) becomes the switch turning rate function given by (26). In the next section we investigate the existence of travelling wave solutions under this specific turning rate. 
4.3 Travelling waves for the switch turning rate (26)

For $\lambda$ given by (26), $\lambda_{1}$ and $\lambda_{2}$ are given by

$$
\lambda_{1}(\zeta)=\left\{\begin{array}{r}
-2 \lambda_{0}, \quad \zeta<0 \\
0, \quad \zeta=0 \\
2 \lambda_{0}, \quad \zeta>0
\end{array} \quad, \quad \lambda_{2}(\zeta) \equiv 2 \lambda_{0} .\right.
$$

From the numerical investigation, we speculate that there exist travelling wave solutions that have a single wave peak of $S$ and $n$ in both cases I and II for certain parameters, and in the following we seek to prove this conjecture under the assumption $D_{S}=D_{F}=0$ by constructing the solutions. Let us denote

$$
Y_{S}=\left\{f \in C^{1}(\mathbb{R}) ; f \text { is monotonically decreasing for } \xi>0 \text { and increasing for } \xi<0, \lim _{\xi \rightarrow \infty} f(\xi)=0\right\} .
$$

We translate the coordinate system as necessary such that $S$ peaks at $\xi=0$, i.e., arg $\max _{\xi \in \mathbb{R}} S(\xi)=0$. Notice that using the turning kernel (26), the equations (35)-(37) for $\mathcal{N}, \mathcal{N}_{z}$ and $\mathcal{J}_{z}$ only depend on the sign of $\mathcal{S}^{\prime}$. Therefore assuming $\mathcal{S} \in Y_{S}$, Equations (35)-(37) become

$$
\begin{aligned}
\mathcal{N}^{\prime} & =-\sigma_{2} \mathcal{N}, \\
m^{\prime} & =-A m+\mathcal{F} \mathcal{N} a,
\end{aligned}
$$

for $\xi>0\left(\mathcal{S}^{\prime}<0\right.$ therefore $\left.\lambda_{1}=-2 \lambda_{0}\right)$ and

$$
\begin{aligned}
\mathcal{N}^{\prime} & =\sigma_{1} \mathcal{N}, \\
m^{\prime} & =B m+\mathcal{F} \mathcal{N} a,
\end{aligned}
$$

for $\xi<0\left(\mathcal{S}^{\prime}>0\right.$ therefore $\left.\lambda_{1}=2 \lambda_{0}\right)$. Here

$$
\sigma_{1}=\frac{2 \lambda_{0}}{s+c}, \quad \sigma_{2}=\frac{2 \lambda_{0}}{s-c}, \quad m=\left(\begin{array}{c}
\mathcal{N}_{z} \\
\mathcal{J}_{z}
\end{array}\right),
$$

and

$$
\begin{aligned}
A & =\frac{1}{s^{2}-c^{2}}\left(\begin{array}{cc}
2 \lambda_{0} s+c t_{m}^{-1} & 2 \lambda_{0}+t_{m}^{-1} \\
2 \lambda_{0} c s+s^{2} t_{m}^{-1} & 2 \lambda_{0} c+c t_{m}^{-1}
\end{array}\right), \quad a=\frac{1}{t_{m}\left(s^{2}-c^{2}\right)}\left(\begin{array}{c}
2 c \\
s^{2}+c^{2}
\end{array}\right), \\
B & =\frac{1}{s^{2}-c^{2}}\left(\begin{array}{cc}
2 \lambda_{0} s-c t_{m}^{-1} & -2 \lambda_{0}-t_{m}^{-1} \\
2 \lambda_{0} c s-s^{2} t_{m}^{-1} & -2 \lambda_{0} c-c t_{m}^{-1}
\end{array}\right) .
\end{aligned}
$$

These equations are linear in $\mathcal{N}, \mathcal{N}_{z}$ and $\mathcal{J}_{z}$ and hence we can solve them in terms of $\mathcal{F}$ by treating the speed $c$ as a parameter. The following Lemma gives the solution to $\mathcal{N}$ and predicts waves with a sharp wavefront and a shallower tail, consistent with the simulations in Section 3.

Lemma 2 Assume the system (28)-(33) has traveling wave solutions with a single peak of $S$, then $\mathcal{N}$ is of the form

$$
\mathcal{N}(\xi)= \begin{cases}n_{0} e^{\sigma_{1} \xi}, & \xi \leq 0 \\ n_{0} e^{-\sigma_{2} \xi}, & \xi>0\end{cases}
$$

where $n_{0}=\mathcal{N}(0)$.

Proof. This follows directly from integrating equations (43) and (45).

In the following we determine the existence of traveling wave solutions of the two types and the speed of the wave as a function of the model parameters under the assumption $D=0$. 
4.3.1 Case I: Travelling waves without starvation. In this section, we state the main result for the existence of the no-starvation traveling wave solutions, that is, $\min F=F_{-}>z_{c}$ where $z_{c}$ is the switching threshold in the function $h$ given by (12). Hence we obtain $\mathcal{H}=\mathcal{N}$, and by assuming $D=0$, (38)-(39) read

$$
\begin{aligned}
& -c \mathcal{S}^{\prime}=\alpha \mathcal{F N}-\gamma \mathcal{S}, \\
& -c \mathcal{F}^{\prime}=-\beta \mathcal{F N} .
\end{aligned}
$$

We show that a nonzero linear degradation of the attractant is necessary for the existence of a travelling wave solution. We define the auxiliary function

$$
I(\theta, z)=z e^{\theta z}(\theta z)^{-(1+z)} \int_{0}^{\theta z} \eta^{z} e^{-\eta} \mathrm{d} \eta .
$$

It can be written as

$$
I(\theta, z)=z e^{\theta z}(\theta z)^{-(1+z)} \bar{\gamma}(z+1, \theta z)
$$

where $\bar{\gamma}(z+1, \theta z)$ is the so called lower incomplete gamma function which is defined by

$$
\bar{\gamma}\left(q_{1}, q_{2}\right)=\int_{0}^{q_{2}} \eta^{q_{1}-1} e^{-\eta} \mathrm{d} \eta .
$$

Theorem 3 Assume $D_{S}=D_{F}=0$, and assume $h, \lambda$ are given by (12), (26), then

(i) If $\gamma=0$, then there does not exist traveling wave solution with $\min F=F_{-}>z_{c}$ and $\mathcal{S} \in Y_{S}$.

(ii) If $\gamma>0$, then there exist a traveling wave solution with $\min F=F_{-}>z_{c}$ and $\mathcal{S} \in Y_{S}$ if and only if there exists $\bar{\tau}_{2}$ such that

$$
I\left(\beta / \gamma, \bar{\tau}_{2}\right)=1, \quad \bar{\tau}_{2}>\gamma / \beta
$$

and $F_{+}$such that

$$
F_{+} \exp \left[-\beta / \lambda_{0}-2 \bar{\tau}_{2} \beta / \gamma\right]>z_{c}
$$

Furthermore the speed of the traveling wave is uniquely determined as

$$
c=\frac{s \gamma}{\gamma+2 \lambda_{0} \overline{\tau_{2}}}
$$

and the traveling wave solution $\mathcal{N}, \mathcal{S}$ and $\mathcal{F}$ are explicitly given by (47), and

$$
S= \begin{cases}S_{0} e^{\frac{\gamma}{c} \xi}\left(1-\tau_{1} e^{-t_{1}} t_{1}^{-1+\tau_{1}} \int_{t_{1}}^{t_{1} e^{\sigma_{1} \xi}} \eta^{-\tau_{1}} e^{\eta} d \eta\right), & \xi \leq 0, \\ S_{0} e^{\frac{\gamma}{c} \xi}\left(1+\tau_{2} e^{t_{2}} t_{2}^{-1-\tau_{2}} \int_{t_{2}}^{t_{2} e^{-\sigma_{2} \xi}} \eta^{\tau_{2}} e^{-\eta} d \eta\right), & \xi>0\end{cases}
$$

with $S_{0}=\alpha F_{0} / \gamma$,

$$
\mathcal{F}= \begin{cases}F_{0} \exp \left[t_{1}\left(e^{\sigma_{1} \xi}-1\right)\right], & \xi \leq 0 \\ F_{0} \exp \left[t_{2}\left(1-e^{-\sigma_{2} \xi}\right)\right], & \xi>0\end{cases}
$$

A sufficient condition for the existence of $\overline{\tau_{2}}$ is

$$
I\left(\theta, \frac{1}{\theta}\right)=\frac{e}{\theta} \int_{0}^{1} \eta^{\frac{1}{\theta}} e^{-\eta} d \eta<1, \text { with } \theta=\beta / \gamma .
$$

For the integrity of the main text, we give the proof and formulae in Appendix B, while a numerical illustration is given by Figure 5. We note that the speed of the travelling wave solution is determined by the parameters $\beta$ and $\gamma$ : other parameters affect only the shape of the solution. We also note that the width of the traveling band is on a spacial scale of millimeters, which agrees with the experimental findings $[9,10]$. 

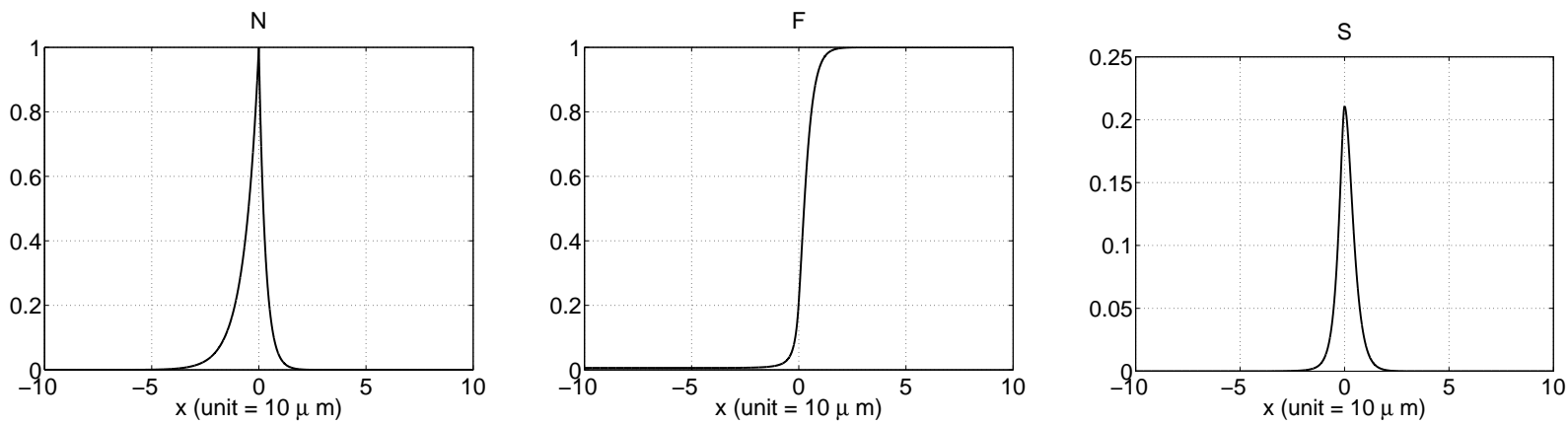

Fig. 5 Travelling waves under no starvation. The wave speed $c$ is given by $c / s=0.3910$ while other parameters were set at $D_{S}=D_{F}=0 \mathrm{~cm}^{2} / \mathrm{sec}, \beta=2 / \mathrm{sec}, \alpha=2 / \mathrm{sec}, \gamma=2 / \mathrm{sec}, \lambda_{0}=10 / \mathrm{sec}$ and $\mathrm{s}=1 \mu \mathrm{m} / \mathrm{sec}$. The $x$-axis is scaled by $s \lambda_{0}=10 \mu \mathrm{m}$.

4.3.2 Case II: Travelling waves with starvation. We seek travelling wave solutions for the case in which succinate-depleted cells convert to aspartate consumption. Here we focus on the case $\gamma=0$ and $h(z)=z$ as employed in the numerics. We note that this case is more complicated since it is also necessary to solve the equations for $\mathcal{N}_{z}$ and $\mathcal{J}_{z}$. As for Case I, we assume $D=0$ in the following analysis. The system (38)-(39) is

$$
\begin{aligned}
\mathcal{S}^{\prime} & =-\frac{\alpha}{c} \mathcal{F} \mathcal{N}_{z}+\frac{\beta}{c}\left(\mathcal{N}-\mathcal{N}_{z}\right) \mathcal{S}, \\
\mathcal{F}^{\prime} & =\frac{\beta}{c} \mathcal{F N}_{z} .
\end{aligned}
$$

Therefore the system (43), (44), (45), (46), (56) and (57) is a closed system of five equations for five unknowns $\mathcal{N}, \mathcal{N}_{z}, \mathcal{J}_{z}, \mathcal{S}$ and $\mathcal{F}$. In the following we first prove in Theorem 4 that given $\mathcal{S} \in Y_{S}$, the subsystem (43), (44), (45), (46) and (57) has a unique solution satisfying the boundary conditions (40) and give the forms of $\mathcal{N}, \mathcal{N}_{z}, \mathcal{J}_{z}, \mathcal{F}$ in terms of $\mathcal{S}$, then we show that $\mathcal{S} \in Y_{S}$. Since the form for $\mathcal{S}$ and $\mathcal{S}^{\prime \prime}$ are much more complicated than Case I, we only show numerically that $\mathcal{S} \in Y_{S}$ in Figure 6.

Theorem 4 Assume $\mathcal{S} \in Y_{S}$, treating c as a parameter, then the system (43), (44), (45), (46), and (57) has a unique bounded solution that satisfies the boundary conditions (40) with $\mathcal{N}(0)=n_{0}, \mathcal{F}(0)=\mathcal{F} \mathcal{F}_{0}$ and $\mathcal{F}$ monotonically increasing. In addition, if

$$
F_{0} \exp \left[\frac{\beta(s-c)}{2 \lambda_{0} c}\right] \leq 1
$$

then $F_{+}=\lim _{\xi \rightarrow+\infty} \mathcal{F}(\xi) \leq 1$

The proof of Theorem 4 is given in Appendix C.

Given $\mathcal{N}, \mathcal{N}_{z}, \mathcal{J}_{z}$ and $\mathcal{F}$, then $\mathcal{S}$ can be expressed as

$$
\mathcal{S}(\xi)=\exp \left[\frac{\beta}{c} \int_{0}^{\xi}\left(\mathcal{N}-\mathcal{N}_{z}\right) \mathrm{d} \zeta\right]\left(S_{0}-\frac{\alpha}{c} \int_{0}^{\xi} \mathcal{F} \mathcal{N}_{z} \exp \left[-\frac{\beta}{c} \int_{0}^{\zeta}\left(\mathcal{N}-\mathcal{N}_{z}\right) \mathrm{d} \eta\right] \mathrm{d} \zeta\right)
$$

with

$$
\mathcal{S}_{0}=\frac{\alpha \mathcal{F}_{0} \mathcal{N}_{z}(0)}{\beta\left(n_{0}-\mathcal{N}_{z}(0)\right)}
$$

determined by $\mathcal{S}^{\prime}(0)=0$. From the expression we see that $\mathcal{S}$ is bounded in $\xi \in \mathbb{R}$ given (58). To prove that the solutions given in Theorem 4 can be extended to a traveling wave solution with starvation by this 
formula, we need to determine $c$ with $0<c<s$ such that $\mathcal{S}^{\prime \prime} \in Y_{S}$ and $\mathcal{S}^{\prime}(\xi) \neq 0$ for $\xi \neq 0$ (similar to the argument in the proof of Theorem 3). When $\mathcal{S}^{\prime}=0$, we have

$$
\begin{aligned}
\mathcal{S}^{\prime \prime} & =-\frac{\alpha}{c} \mathcal{F}^{\prime} \mathcal{N}_{z}-\frac{\alpha}{c} \mathcal{F} \mathcal{N}_{z}^{\prime}+\frac{\beta}{c}\left(\mathcal{N}^{\prime}-\mathcal{N}_{z}^{\prime}\right) \mathcal{S} \\
& = \begin{cases}-\frac{\alpha \beta}{c^{2}} \mathcal{F} \mathcal{N}_{z}^{2}-\frac{\alpha}{c} \mathcal{F} \mathcal{N}_{z}^{\prime}+\frac{\beta}{c} \mathcal{S}\left(-\sigma_{2} \mathcal{N}-\mathcal{N}_{z}^{\prime}\right) & \xi>0 \\
-\frac{\alpha \beta}{c^{2}} \mathcal{F} \mathcal{N}_{z}^{2}-\frac{\alpha}{c} \mathcal{F} \mathcal{N}_{z}^{\prime}+\frac{\beta}{c} \mathcal{S}\left(\sigma_{1} \mathcal{N}-\mathcal{N}_{z}^{\prime}\right) & \xi<0 .\end{cases}
\end{aligned}
$$

The form of $\mathcal{S}^{\prime \prime}$ in this case makes the analysis of the sign of $\mathcal{S}^{\prime \prime}$ rather complicated, but we show the resulting wave through a numerical example in Figure 6. We note that the width of the traveling band is on a spacial scale of millimeters, which agrees with the experimental findings $[9,10]$. To generate the waves, first, for each $c$, we numerically solve $\mathcal{N}, \mathcal{N}_{z}, \mathcal{J}_{z}$ and $\mathcal{F}$ by iteration using the mapping $W$ defined in the proof of Theorem 4 (since the mapping is a contraction, the iteration method converges). We then increase $c$ from 0 to $s$ in small increments to find $c^{*}$ such that $\lim _{\xi \rightarrow \infty} \mathcal{S}=\mathcal{S}_{+}=0$.
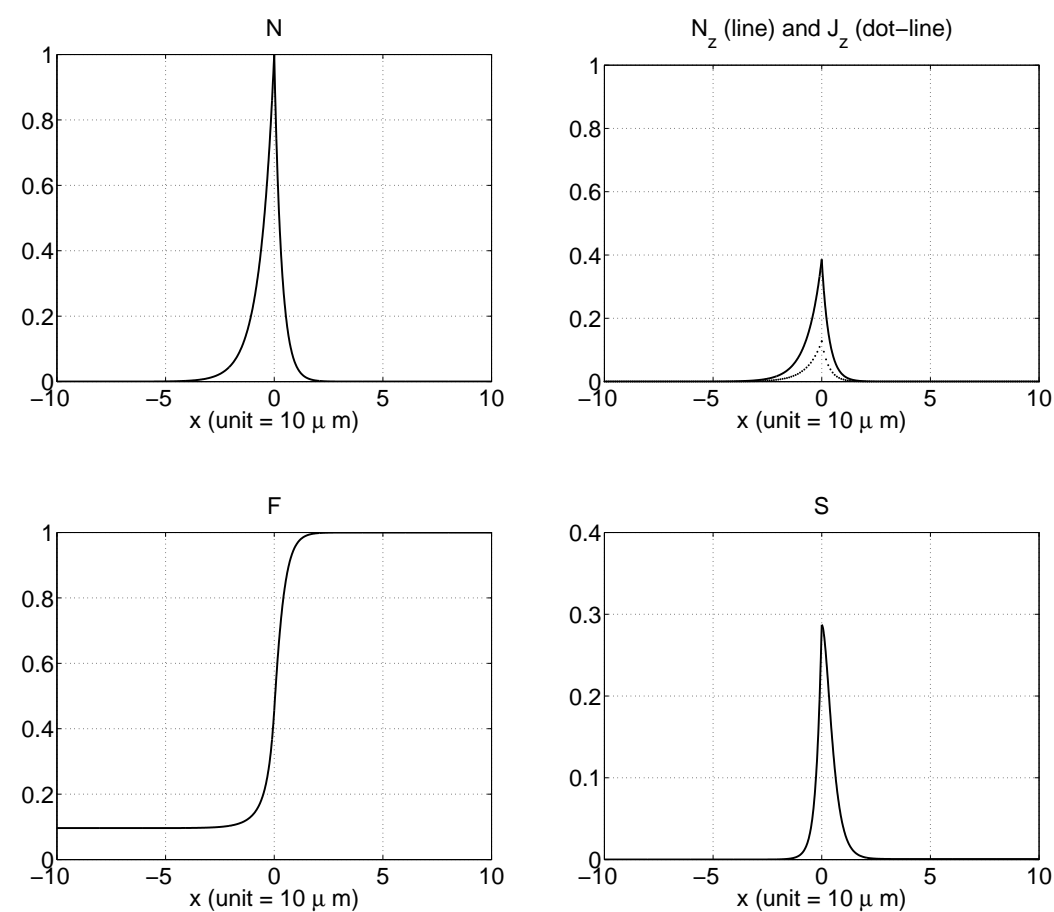

Fig. 6 Travelling waves with speed $c / s=0.33$ of case II (i.e., including starvation). Parameters used $t_{m}=10 \mathrm{~min}$, others are the same as in Figure 5. The wave is constructed using the iterative method described in the text. Numerical simulations show that the wave speed is uniquely determined by $F_{+}$and $S_{+}$. The $x$-axis is scaled by $s \lambda_{0}=10 \mu \mathrm{m}$.

\section{Discussion}

In this paper we have formulated a mathematical model for bacterial chemotaxis that takes into account a variety of internal processes, such as details of signal transduction and the metabolic response of cells to low nutrient levels. We demonstrated that the model is well-posed by proving global existence under the special case of zero cell growth. Numerical simulations of a further reduced model implied that, under certain limiting scenarios, travelling wave behaviour could occur. The existence of these travelling waves was demonstrated under two specific cases. 
As described in the introduction, classical chemotaxis models of the type studied in [23] typically requires singularities in the chemotactic sensitivity to generate travelling waves under scenarios of zero cellular growth: such an assumption provides the cells left in the wake of a travelling band unbounded velocities, allowing them to keep pace with the spreading band. In the hyperbolic model developed here, travelling waves have been mathematically demonstrated even for bounded velocities and turning rates. We should note that we require the assumption in which the turning rate function of the cells allows them to respond to infinitesimally shallow chemotactic gradients, in order to keep the band undamped over time. The question of whether this property can be relaxed under more complicated versions of the model remains unanswered. From a biological perspective, it is worth noting that the numerical simulations performed here for more realistic turning rate forms and biologically relevant parameter values display relatively slow decay of the bacterial bands on experimentally relevant timescales.

To address the fundamental question of travelling waves driven by chemotaxis, the modelling here has excluded (explicit) inclusion of bacteria proliferation. Obviously, in the application to experimental observations, growth is a significant factor both during the propagation of the swarm front as well as any patterning processes occurring in the wake of the front. Through analogy with classical chemotaxis models and the analysis in [25], it is highly plausible that the addition of indirect nutrient-dependent growth rate $k(\mathbf{z})$ into the hyperbolic model probably can lead to travelling waves under less stringent requirements for the turning rate functions. A study into this important case is left for future investigations. Recently, it has been shown that aggregates of bacteria may show "volcano-effect", where bacteria regularly overshoot a sharp peak of chemoattractant [30]. This phenomena was not observed in the analysis of the simplified model (18)-(21) since we assumed the temporal signal sensing as effective as spatial signal sensing. Whether traveling wave solutions with observable "volcano effect" exist for the full model (8)-(11) is left for future investigations.

Acknowledgements: CX is supported by US NSF Award 0635561. KJP would like to acknowledge support from the Mathematical Biosciences Institute and BBSRC grant BB/D019621/1 for the Centre for Systems Biology at Edinburgh. HJH is supported by Priority Research Centers Program through the National Research Foundation of Korea (NRF) funded by the Ministry of Education, Science and Technology (Grant 2009-0094068). RE is supported by the European Research Council Starting Independent Researcher Grant. This publication is based on work supported by Award No. KUK-C1-013-04, made by King Abdullah University of Science and Technology (KAUST). RE would also like to thank Somerville College, University of Oxford, for Fulford Junior Research Fellowship.

\section{A Appendix: Proofs of Theorems 1 and 2}

To prove Theorems 1, we need estimates on growth in time of $S, F$ in $L^{\infty}$ norm, which essentially control time evolution of $L^{p}$ norm of $p^{+}$and $p^{-}$by applying Lemma 3.1 from [22], i.e. the Gronwall inequality which ensures boundedness in time in $L^{p}$ norm of solutions to our kinetic model. Let

$$
n(x, t)=\int_{\mathbb{Y}} \int_{\mathbb{Z}} p^{+}(x, \mathbf{y}, \mathbf{z})+p^{-}(x, \mathbf{y}, \mathbf{z}) \mathrm{d} \mathbf{y} \mathrm{d} \mathbf{z},
$$

where $\mathbb{Y}=\mathbb{R}^{q}, \mathbb{Z}=\mathbb{R}^{2}$ in the notation of Theorem 1 and $\mathbb{Y}=\emptyset, \mathbb{Z}=\mathbb{R}$ for Theorem 2 , respectively. Note that this definition for $n(x, t)$ is the same as the one defined in Section 4.

First, we prove the following auxiliary lemma.

Lemma 3 If $n \in L^{\infty}\left([0, \infty): L^{1}(\mathbb{R})\right)$, then the solution $(S, F)$ in $(10)-(11)$ satisfies

$$
\begin{gathered}
\|F(t)\|_{L^{\infty}(\mathbb{R})} \leq C\left\|F_{0}\right\|_{L^{\infty}(\mathbb{R})}, \\
\|S(t)\|_{L^{\infty}(\mathbb{R})} \leq C\left(\left\|F_{0}\right\|_{L^{\infty}(\mathbb{R})}\left\|n_{0}\right\|_{L^{1}(\mathbb{R})}+\left\|S_{0}\right\|_{L^{\infty}(\mathbb{R})}\right),
\end{gathered}
$$

where $C$ depends on $\alpha, \beta, \gamma, D_{F}$ and $D_{S}$ and where $n_{0}=\int_{\mathbb{Y}} \int_{\mathbb{Z}} p_{0}^{+}+p_{0}^{-} d \mathbf{y} d \mathbf{z}$.

Proof. Since the proof is similarly given as in the first part of the proof of Lemma 4, we omit it.

Now we give a brief sketch of the proof of Theorem 1 


\section{Proof (of Theorem 1)}

Integrating (8) and (9) with $k(z)=0$ over $\mathbb{R} \times \mathbb{Y} \times \mathbb{Z}$, and noticing that $p^{ \pm}$vanishes for large $\mathbf{y}$ and $\mathbf{z}$ by

(5) - (7) and Lemma 3 , we obtain that $p^{+}(\cdot, \cdot, \cdot, t) \in L^{1}\left(\mathbb{R} \times \mathbb{R}^{q} \times \mathbb{R}^{2}\right)$.

Given any $1<r<\infty$, by multiplying $r\left(p^{+}\right)^{r-1}$ to (8), one obtains,

$$
\begin{aligned}
\frac{\partial}{\partial t}\left[\left(p^{+}\right)^{r}\right]+s \frac{\partial}{\partial x}\left[\left(p^{+}\right)^{r}\right]+r\left(p^{+}\right)^{r-1} \sum_{i=1}^{q} \frac{\partial}{\partial y_{i}}\left[f_{i} p^{+}\right] & +r\left(p^{+}\right)^{r-1} \sum_{i=1}^{2} \frac{\partial}{\partial z_{i}}\left[g_{i} p^{+}\right] \\
& =\lambda(\mathbf{y})\left[-r\left(p^{+}\right)^{r}+r\left(p^{+}\right)^{r-1} p^{-}\right] .
\end{aligned}
$$

Integrating the above equation over $\mathbb{R} \times \mathbb{Y} \times \mathbb{Z}$, one obtains,

$$
\begin{gathered}
\frac{\mathrm{d}}{\mathrm{d} t}\left\|p^{+}\right\|_{L^{r}(\mathbb{R} \times \mathbb{Y} \times \mathbb{Z})}^{r}+\int r\left(p^{+}\right)^{r-1} \sum_{i=1}^{q} \frac{\partial}{\partial y_{i}}\left[f_{i} p^{+}\right] \mathrm{d} x \mathrm{~d} \mathbf{y} \mathrm{d} \mathbf{z}+\int r\left(p^{+}\right)^{r-1} \sum_{i=1}^{2} \frac{\partial}{\partial z_{i}}\left[g_{i} p^{+}\right] \mathrm{d} x \mathrm{~d} \mathbf{y d} \mathbf{z} \\
=-r \int \lambda(\mathbf{y})\left(p^{+}\right)^{r} \mathrm{~d} x \mathrm{~d} \mathbf{y} \mathrm{d} \mathbf{z}+r \int \lambda(\mathbf{y})\left(p^{+}\right)^{r-1} p^{-} \mathrm{d} x \mathrm{~d} \mathbf{y} \mathrm{d} \mathbf{z} .
\end{gathered}
$$

Since

$$
\begin{aligned}
\frac{\partial}{\partial y_{i}}\left[f_{i}\left(p^{+}\right)^{r}\right] & =\frac{\partial f_{i}}{\partial y_{i}}\left(p^{+}\right)^{r}+r\left(p^{+}\right)^{r-1} f_{i} \frac{\partial p^{+}}{\partial y_{i}} \\
& =\frac{\partial f_{i}}{\partial y_{i}}\left(p^{+}\right)^{r}+r\left(p^{+}\right)^{r-1} \frac{\partial}{\partial y_{i}}\left[f_{i} p^{+}\right]-r \frac{\partial f_{i}}{\partial y_{i}}\left(p^{+}\right)^{r} \\
& =(1-r) \frac{\partial f_{i}}{\partial y_{i}}\left(p^{+}\right)^{r}+r\left(p^{+}\right)^{r-1} \frac{\partial}{\partial y_{i}}\left[f_{i} p^{+}\right]
\end{aligned}
$$

one obtains

$$
r\left(p^{+}\right)^{r-1} \frac{\partial}{\partial y_{i}}\left[f_{i} p^{+}\right]=\frac{\partial}{\partial y_{i}}\left[f_{i}\left(p^{+}\right)^{r}\right]+(r-1) \frac{\partial f_{i}}{\partial y_{i}}\left(p^{+}\right)^{r},
$$

summing over $i$, it becomes

$$
r\left(p^{+}\right)^{r-1} \sum_{i=1}^{q} \frac{\partial}{\partial y_{i}}\left[f_{i} p^{+}\right]=\nabla_{\mathbf{y}} \cdot\left(\mathbf{f}\left(p^{+}\right)^{r}\right)+(r-1)\left(\nabla_{\mathbf{y}} \cdot \mathbf{f}\right)\left(p^{+}\right)^{r} .
$$

Integrating over $\mathbb{R} \times \mathbb{Y} \times \mathbb{Z}$, and noticing the boundary terms all vanish, one obtains,

$$
\int r\left(p^{+}\right)^{r-1} \sum_{i=1}^{q} \frac{\partial}{\partial y_{i}}\left[f_{i} p^{+}\right] \mathrm{d} x \mathrm{~d} \mathbf{y} \mathrm{d} \mathbf{z}=(r-1) \int\left(\nabla_{\mathbf{y}} \cdot \mathbf{f}\right)\left(p^{+}\right)^{r} \mathrm{~d} x \mathrm{~d} \mathbf{y} \mathrm{d} \mathbf{z} .
$$

Similarly,

$$
\int r\left(p^{+}\right)^{r-1} \sum_{i=1}^{2} \frac{\partial}{\partial z_{i}}\left[g_{i} p^{+}\right] \mathrm{d} x \mathrm{~d} \mathbf{y} \mathrm{d} \mathbf{z}=(r-1) \int\left(\nabla_{\mathbf{z}} \cdot \mathbf{g}\right)\left(p^{+}\right)^{r} \mathrm{~d} x \mathrm{~d} \mathbf{y} \mathrm{d} \mathbf{z} .
$$

By Young's Inequality, one has

$$
\left(p^{+}\right)^{r-1} p^{-} \leq \frac{r-1}{r}\left(p^{+}\right)^{r}+\frac{1}{r}\left(p^{-}\right)^{r}
$$

therefore

$$
r \int \lambda(\mathbf{y})\left(p^{+}\right)^{r-1} p^{-} \mathrm{d} x \mathrm{~d} \mathbf{y} \mathrm{d} \mathbf{z} \leq(r-1) \int \lambda(\mathbf{y})\left(p^{+}\right)^{r} \mathrm{~d} x \mathrm{~d} \mathbf{y} \mathrm{d} \mathbf{z}+\int \lambda(\mathbf{y})\left(p^{-}\right)^{r} \mathrm{~d} x \mathrm{~d} \mathbf{y} \mathrm{d} \mathbf{z},
$$

and

$$
\text { RHS of }(64) \leq \int \lambda(\mathbf{y})\left[-\left(p^{+}\right)^{r}+\left(p^{-}\right)^{r}\right] \mathrm{d} x \mathrm{~d} \mathbf{y} \mathrm{d} \mathbf{z}
$$


Considering (65) - (67), from (64) we obtain

$$
\begin{aligned}
\frac{\mathrm{d}}{\mathrm{d} t}\left\|p^{+}\right\|_{L^{r}(\mathbb{R} \times \mathbb{Y} \times \mathbb{Z})}^{r} \leq & -(r-1) \int\left[\nabla_{\mathbf{y}} \cdot \mathbf{f}(\mathbf{y}, S)+\nabla_{\mathbf{z}} \cdot \mathbf{g}(\mathbf{z}, F)\right]\left(p^{+}\right)^{r} \mathrm{~d} x \mathrm{~d} \mathbf{y d} \mathbf{z} \\
& +\int \lambda(\mathbf{y})\left[-\left(p^{+}\right)^{r}+\left(p^{-}\right)^{r}\right] \mathrm{d} x \mathrm{~d} \mathbf{y} \mathrm{d} \mathbf{z} .
\end{aligned}
$$

Similarly, by following the same procedure for (9), one obtains,

$$
\begin{aligned}
\frac{\mathrm{d}}{\mathrm{d} t}\left\|p^{-}\right\|_{L^{r}(\mathbb{R} \times \mathbb{Y} \times \mathbb{Z})}^{r} \leq & -(r-1) \int\left[\nabla_{\mathbf{y}} \cdot \mathbf{f}(\mathbf{y}, S)+\nabla_{\mathbf{z}} \cdot \mathbf{g}(\mathbf{z}, F)\right]\left(p^{-}\right)^{r} \mathrm{~d} x \mathrm{~d} \mathbf{y d} \mathbf{z} \\
& +\int \lambda(\mathbf{y})\left[\left(p^{+}\right)^{r}-\left(p^{-}\right)^{r}\right] \mathrm{d} x \mathrm{~d} \mathbf{y d} \mathbf{z} .
\end{aligned}
$$

Adding (68) and (69), and using assumptions (15) and Lemma 3, one obtains

$$
\begin{aligned}
\frac{\mathrm{d}}{\mathrm{d} t}\left(\| p^{+}\right. & \left.\left\|_{L^{r}(\mathbb{R} \times \mathbb{Y} \times \mathbb{Z})}^{r}+\right\| p^{-} \|_{L^{r}(\mathbb{R} \times \mathbb{Y} \times \mathbb{Z})}^{r}\right) \\
& \leq-(r-1) \int\left[\nabla_{\mathbf{y}} \cdot \mathbf{f}(\mathbf{y}, S)+\nabla_{\mathbf{z}} \cdot \mathbf{g}(\mathbf{z}, F)\right]\left[\left(p^{-}\right)^{r}+\left(p^{-}\right)^{r}\right] \mathrm{d} x \mathrm{~d} \mathbf{y} \mathrm{d} \mathbf{z} \\
& \leq(r-1)\left(\Pi_{1}\left(\|S(\cdot, t)\|_{L^{\infty}}\right)+\Pi_{2}\left(\|F(\cdot, t)\|_{L^{\infty}}\right)\right)\left(\left\|p^{+}\right\|_{L^{r}(\mathbb{R} \times \mathbb{Y} \times \mathbb{Z})}^{r}+\left\|p^{-}\right\|_{L^{r}(\mathbb{R} \times \mathbb{Y} \times \mathbb{Z})}^{r}\right) \\
& \leq C(r-1)\left(\left\|p^{+}\right\|_{L^{r}(\mathbb{R} \times \mathbb{Y} \times \mathbb{Z})}^{r}+\left\|p^{-}\right\|_{L^{r}(\mathbb{R} \times \mathbb{Y} \times \mathbb{Z})}^{r}\right)
\end{aligned}
$$

where $C$ is independent of $r$ and depends on $\left\|S_{0}\right\|_{L^{\infty}},\|F(\cdot, t)\|_{L^{\infty}},\left\|n_{0}\right\|_{L^{1}(\mathbb{R})}$. Applying the Gronwall's inequality to the above inequality, one obtains,

$$
\left\|p^{ \pm}(t)\right\|_{L^{r}(\mathbb{R} \times \mathbb{Y} \times \mathbb{Z})}^{r} \leq H_{0}(r) e^{C(r-1) t},
$$

with

$$
H_{0}(r)=\left\|p^{+}(0)\right\|_{L^{r}(\mathbb{R} \times \mathbb{Y} \times \mathbb{Z})}^{r}+\left\|p^{-}(0)\right\|_{L^{r}(\mathbb{R} \times \mathbb{Y} \times \mathbb{Z})}^{r} .
$$

Since $H_{0}(r)^{1 / r}$ is bounded in $r \in(1, \infty)$, we obtain from $(70)$ that

$$
\left\|p^{ \pm}(t)\right\|_{L^{r}(\mathbb{R} \times \mathbb{Y} \times \mathbb{Z})} \leq C e^{C t(r-1) / r} \leq C e^{C t},
$$

where $C$ are constants that depend only on the initial data but not $r$. By taking the limit as $r \rightarrow \infty$, one obtains

$$
p^{ \pm}(\cdot, \cdot, \cdot, t) \in L^{\infty}(\mathbb{R} \times \mathbb{Y} \times \mathbb{Z})
$$

Once we have a priori estimates, we can apply standard methods such as a vanishing viscosity method as in [22] to obtain a global existence of solutions. Thus we complete a sketch of the proof of the theorem.

To prove Theorems 2, we need estimates of not only $S, F$ but also their derivatives in $L^{\infty}$ norm. For the purpose, we give the following lemma.

Lemma 4 If $n \in L^{\infty}\left([0, \infty): L^{1}(\mathbb{R}) \cap L^{2}(\mathbb{R})\right)$, then the solution $(S, F)$ in (10) satisfies

$$
\begin{gathered}
\|F(t)\|_{L^{r}(\mathbb{R})} \leq C_{1}\left\|F_{0}\right\|_{L^{r}(\mathbb{R})}, \quad 1 \leq r \leq \infty, \\
\|S(t)\|_{L^{r}(\mathbb{R})} \leq C_{1}\left(\left\|F_{0}\right\|_{L^{\infty}(\mathbb{R})}\left\|n_{0}\right\|_{L^{1}(\mathbb{R})} t^{\frac{1}{r}}+\left\|S_{0}\right\|_{L^{r}(\mathbb{R})}\right), \quad 1 \leq r \leq \infty, \\
\left\|\frac{\partial F}{\partial x}(t)\right\|_{L^{\infty}(\mathbb{R})} \leq C_{2}\left[1+\|n(0)\|_{L^{1}(\mathbb{R})}\left\{1+\ln \left(\|n(t)\|_{L^{2}(\mathbb{R})}+1\right)\right\}\right], \\
\left\|\frac{\partial S}{\partial x}(t)\right\|_{L^{\infty}(\mathbb{R})} \leq C_{2}\left[1+\|n(0)\|_{L^{1}(\mathbb{R})}\left\{1+\ln \left(\|n(t)\|_{L^{2}(\mathbb{R})}+1\right)\right\}\right],
\end{gathered}
$$

where $C_{1}$ depends on $\alpha, \beta, \gamma, D_{F}$, and $D_{S}$ and $C_{2}$ depends on $\left\|F_{0}\right\|_{L^{\infty}(\mathbb{R})},\left\|F_{0}\right\|_{L^{2}(\mathbb{R})},\left\|\frac{\partial F_{0}}{\partial x}\right\|_{L^{\infty}(\mathbb{R})},\left\|S_{0}\right\|_{L^{\infty}(\mathbb{R})}$, $\left\|S_{0}\right\|_{L^{2}(\mathbb{R})},\left\|\frac{\partial S_{0}}{\partial x}\right\|_{L^{\infty}(\mathbb{R})}$. 
Proof. We give only estimates for $F$ and $\frac{\partial F}{\partial x}$ and note that the estimates for $S$ and $\frac{\partial S}{\partial x}$ can be similarly derived. For simplicity, we assume $D_{F}=1$ and $\beta=1$. First, we integrate (21) to get (72) for $r=1$. For $r \geq 2$, we multiply (21) by $p F^{p-1}$, integrate with respect to $x$, and use $F \geq 0$ to derive (72) for $2 \leq r<\infty$. And by taking $r \rightarrow \infty$, we can obtain (21) for $r=\infty$.

Note that the heat kernel $\mathcal{H}$ of the heat operator is given by

$$
\mathcal{H}(x, t)=\frac{1}{\sqrt{t}} \exp \left(-\frac{x^{2}}{4 t}\right)
$$

and its Fourier transform is

$$
\hat{\mathcal{H}}(\xi, t)=\exp \left(-4 t \xi^{2}\right) .
$$

We apply Duhamel's principle to obtain

$$
\begin{aligned}
F(x, t) & =\mathcal{H} * F_{0}-\int_{0}^{t}\left(\mathcal{H} *\left[F \int_{\mathbb{Y}} \int_{\mathbb{Z}} h\left(z_{2}\right)\left[p^{+}(x, \mathbf{y}, \mathbf{z})+p^{-}(x, \mathbf{y}, \mathbf{z})\right] \mathrm{d} \mathbf{y} \mathrm{d} \mathbf{z}\right]\right)(x, t-\tau) \mathrm{d} \tau \\
& =\mathcal{H} * F_{0}-\int_{0}^{t}(\mathcal{H} *[F H])(x, t-\tau) \mathrm{d} \tau,
\end{aligned}
$$

where we define

$$
H=\int_{\mathbb{Y}} \int_{\mathbb{Z}} h\left(z_{2}\right)\left[p^{+}(x, \mathbf{y}, \mathbf{z})+p^{-}(x, \mathbf{y}, \mathbf{z})\right] \mathrm{d} \mathbf{y} \mathrm{d} \mathbf{z},
$$

which is the same as $H$ in Section 4 . Note that

$$
\|H\|_{L^{r}(\mathbb{R})} \leq\|n\|_{L^{r}(\mathbb{R})} \quad \text { for all } \quad 1 \leq r \leq \infty,
$$

We also have $\sup _{0 \leq \tau \leq t}\|n\|_{L^{1}(\mathbb{R})}=\|n(0)\|_{L^{1}(\mathbb{R})}$ since the total population size is preserved, namely

$$
\int_{\mathbb{R}} n(x, t) d x=\int_{\mathbb{R}} n(0)(x) d x=\int_{\mathbb{R}}\left(n^{+}(0)+n^{-}(0)\right)(x) \mathrm{d} x \quad \text { for all } t .
$$

since, from the equations, we have

$$
\frac{\mathrm{d}}{\mathrm{d} t} \int_{\mathbb{R}} n(x, t) \mathrm{d} x=0 .
$$

Also it can be easily shown that

$$
\|\hat{F H}\|_{L^{\infty}(\mathbb{R})} \leq\|F H\|_{L^{1}(\mathbb{R})} \leq\|F\|_{L^{\infty}(\mathbb{R})}\|H\|_{L^{1}(\mathbb{R})} \leq\left\|F_{0}\right\|_{L^{\infty}(\mathbb{R})}\|n\|_{L^{1}(\mathbb{R})} \leq\left\|F_{0}\right\|_{L^{\infty}(\mathbb{R})}\left\|n_{0}\right\|_{L^{1}(\mathbb{R})},
$$

and

$$
\|\hat{F H}\|_{L^{2}(\mathbb{R})}=\|\hat{F} * \hat{H}\|_{L^{2}(\mathbb{R})} \leq\|\hat{F}\|_{L^{2}(\mathbb{R})}\|\hat{H}\|_{L^{2}(\mathbb{R})}=\|F\|_{L^{2}(\mathbb{R})}\|H\|_{L^{2}(\mathbb{R})} \leq\left\|F_{0}\right\|_{L^{2}(\mathbb{R})}\|n\|_{L^{2}(\mathbb{R})}
$$

Now we estimate $\left\|\frac{\partial F}{\partial x}\right\|_{L^{\infty}(\mathbb{R})}$ as follows:

$$
\begin{aligned}
\left\|\frac{\partial F}{\partial x}\right\|_{L^{\infty}(\mathbb{R})} & \leq C\left\|\frac{\partial F_{0}}{\partial x}\right\|_{L^{\infty}(\mathbb{R})}+\left\|\xi\left(\hat{F}-\mathcal{H} * F_{0}\right)\right\|_{L^{1}(\mathbb{R})} \\
& \leq C\left\|\frac{\partial F_{0}}{\partial x}\right\|_{L^{\infty}(\mathbb{R})}+\int_{-\infty}^{\infty} \int_{0}^{t}|\xi| \hat{\mathcal{H} F} \hat{F} \mid(\xi, t-\tau) \mathrm{d} \tau \mathrm{d} \xi \\
& =C\left\|\frac{\partial F_{0}}{\partial x}\right\|_{L^{\infty}(\mathbb{R})}+\int_{0}^{t} \int_{-\infty}^{\infty}|\xi| \exp \left(-4 \tau \xi^{2}\right)|\hat{F H}(\xi, \tau)| \mathrm{d} \xi \mathrm{d} \tau .
\end{aligned}
$$

The integration above is performed by splitting the time interval into a short time interval $I_{1}$ and a large time interval $I_{2}$ :

$$
\int_{0}^{t} \int_{-\infty}^{\infty}|\xi| \exp \left(-4 \tau \xi^{2}\right)|\hat{F H}(\xi, \tau)| \mathrm{d} \xi \mathrm{d} \tau=I_{1}+I_{2}
$$


where

$$
\begin{aligned}
& I_{1}=\int_{0}^{\kappa} \int_{-\infty}^{\infty}|\xi| \exp \left(-4 \tau \xi^{2}\right)|\hat{F H}(\xi, \tau)| \mathrm{d} \xi \mathrm{d} \tau \\
& I_{2}=\int_{\kappa}^{t} \int_{-\infty}^{\infty}|\xi| \exp \left(-4 \tau \xi^{2}\right)|\hat{F H}(\xi, \tau)| \mathrm{d} \xi \mathrm{d} \tau
\end{aligned}
$$

and where $\kappa>0$ will be chosen later.

Estimation of integral $I_{1}$ :

For $0<\tau<\kappa$, we use Hölder's inequality with $r=r^{\prime}=2$ to obtain:

$$
\begin{gathered}
\int_{-\infty}^{\infty}|\xi| \exp \left(-4 \tau \xi^{2}\right)|\hat{F H}(\xi, \tau)| \mathrm{d} \xi \leq\left(\int_{-\infty}^{\infty} \xi^{2} \exp \left(-8 \tau \xi^{2}\right) \mathrm{d} \xi\right)^{1 / 2}\|\hat{F H}\|_{L^{2}(\mathbb{R})} \\
\leq\left(2 \int_{0}^{\infty} \xi^{2} \exp \left(-8 \tau \xi^{2}\right) \mathrm{d} \xi\right)^{1 / 2}\left\|F_{0}\right\|_{L^{2}(\mathbb{R})}\|n\|_{L^{2}(\mathbb{R})},
\end{gathered}
$$

where we used the Plancherel's equality in $L^{2}$ and (77). Integrating by parts we have

$$
\int_{0}^{\infty} \xi^{2} \exp \left(-8 \tau \xi^{2}\right) \mathrm{d} \xi=\frac{1}{16 \tau} \int_{0}^{\infty} \exp \left(-8 \tau \xi^{2}\right) \mathrm{d} \xi=\frac{\sqrt{\pi}}{64 \sqrt{2}} \tau^{-3 / 2} .
$$

Hence, we obtain

$$
I_{1} \leq \frac{\pi^{1 / 4}}{4 \sqrt[4]{2^{3}}}\left\|F_{0}\right\|_{L^{2}(\mathbb{R})} \sup _{0 \leq \tau \leq t}\|n(\tau)\|_{L^{2}(\mathbb{R})} \int_{0}^{\kappa} \tau^{-3 / 4} \mathrm{~d} \tau \leq \frac{\pi^{1 / 4}}{\sqrt[4]{2^{3}}} \kappa^{1 / 4}\left\|F_{0}\right\|_{L^{2}(\mathbb{R})} \sup _{0 \leq \tau \leq t}\|n\|_{L^{2}(\mathbb{R})} .
$$

Estimation of integral $I_{2}$ :

For $\kappa \leq \tau \leq t$, we use Hölder's inequality with $r=1, r^{\prime}=\infty$ :

$$
\int_{-\infty}^{\infty}|\xi| \exp \left(-8 \tau \xi^{2}\right)|\hat{F H}(\xi, \tau)| \mathrm{d} \xi \leq\|\hat{F H}\|_{L^{\infty}(\mathbb{R})} \int_{-\infty}^{\infty}|\xi| \exp \left(-8 \tau \xi^{2}\right) \mathrm{d} \xi=\frac{1}{4 \tau}\left\|F_{0}\right\|_{L^{\infty}(\mathbb{R})}\left\|n_{0}\right\|_{L^{1}(\mathbb{R})},
$$

where we used (76). So, we have

$$
I_{2} \leq \frac{1}{4}\left\|F_{0}\right\|_{L^{\infty}(\mathbb{R})}\left\|n_{0}\right\|_{L^{1}(\mathbb{R})} \int_{\kappa}^{t} \frac{1}{\tau} \mathrm{d} \tau \leq \frac{1}{4}\left\|F_{0}\right\|_{L^{\infty}(\mathbb{R})}\left\|n_{0}\right\|_{L^{1}(\mathbb{R})}|\ln t-\ln \kappa| .
$$

Therefore, we obtain

$$
\left\|\frac{\partial F}{\partial x}\right\|_{L^{\infty}(\mathbb{R})} \leq C\left\|\frac{\partial F_{0}}{\partial x}\right\|_{L^{\infty}(\mathbb{R})}+C\left(\kappa^{1 / 4}\left\|F_{0}\right\|_{L^{2}(\mathbb{R})} \sup _{0 \leq \tau \leq t}\|n\|_{L^{2}(\mathbb{R})}+\left\|F_{0}\right\|_{L^{\infty}(\mathbb{R})}\left\|n_{0}\right\|_{L^{1}(\mathbb{R})}|\ln t-\ln \kappa|\right) .
$$

We now choose $\kappa>0$ so as to optimize the upper bound for the inequality above:

$$
\kappa=\min \left\{\left(\sup _{0 \leq \tau \leq t}\|n\|_{L^{2}(\mathbb{R})}\right)^{-4}, t\right\} .
$$

Thus we deduce the claim in the lemma:

$$
\begin{aligned}
\left\|\frac{\partial F}{\partial x}\right\|_{L^{\infty}(\mathbb{R})} & \leq C\left\|\frac{\partial F_{0}}{\partial x}\right\|_{L^{\infty}(\mathbb{R})}+C\left[1+(\ln t)_{+}+\left\|F_{0}\right\|_{L^{2}(\mathbb{R})}\left\|F_{0}\right\|_{L^{\infty}(\mathbb{R})}\left\|n_{0}\right\|_{L^{1}(\mathbb{R})}\left|\ln \left(\sup _{0 \leq \tau \leq t}\|n\|_{L^{2}(\mathbb{R})}\right)\right|\right] \\
& \leq C\left(1+(\ln t)_{+}+\left\|n_{0}\right\|_{L^{1}(\mathbb{R})} \sup _{0 \leq \tau \leq t}\left|\ln \left(\|n(\tau)\|_{L^{2}(\mathbb{R})}\right)\right|\right) .
\end{aligned}
$$

Now we give a brief sketch of the proof of Theorem 2 . 
Proof (of Theorem 2) Multiplying $p^{+}$and $p^{-}$to (18) and (19), applying Hölder inequality, and using the assumption (22) and Lemma 4, we obtain

$$
\begin{aligned}
& \frac{\mathrm{d}}{\mathrm{d} t}\left(\left\|p^{+}\right\|_{L^{2}(\mathbb{R} \times \mathbb{R})}^{2}+\left\|p^{-}\right\|_{L^{2}(\mathbb{R} \times \mathbb{R})}^{2}\right) \\
& =-\int_{\mathbb{R} \times \mathbb{R}} \partial_{z}\left(\frac{F-z}{t_{m}}\right)\left[\left(p^{+}\right)^{2}+\left(p^{-}\right)^{2}\right] \mathrm{d} x \mathrm{~d} z \\
& -2 \int_{\mathbb{R} \times \mathbb{R}}\left[\lambda\left(-\frac{\partial S}{\partial x}\right)\left(p^{+}\right)^{2}+\lambda\left(\frac{\partial S}{\partial x}\right)\left(p^{-}\right)^{2}\right] d x d z+2 \int_{\mathbb{R} \times \mathbb{R}}\left[\lambda\left(\frac{\partial S}{\partial x}\right)+\lambda\left(-\frac{\partial S}{\partial x}\right)\right] p^{+} p^{-} \mathrm{d} x \mathrm{~d} z \\
& \leq C\left[1+\left\|\lambda\left(\frac{\partial S}{\partial x}(\cdot, t)\right)\right\|_{L^{\infty}}\right]\left(\left\|p^{+}\right\|_{L^{2}(\mathbb{R} \times \mathbb{R})}^{2}+\left\|p^{-}\right\|_{L^{2}(\mathbb{R} \times \mathbb{R})}^{2}\right) \\
& \leq C\left[1+\left\|\frac{\partial S}{\partial x}(\cdot, t)\right\|_{L^{\infty}}\right]\|n(t)\|_{L^{2}(\mathbb{R})} \\
& \leq C\left[1+\left\|n_{0}\right\|_{L^{1}(\mathbb{R})}\left\{1+\ln \left(\|n(t)\|_{L^{2}(\mathbb{R})}+1\right)\right\}\right]\|n(t)\|_{L^{2}(\mathbb{R})} .
\end{aligned}
$$

Then we use Lemma 3.1 from [22] to deduce Theorem for $r=2$. Once we get the claim for $L^{2}$, we can obtain the boundedness for $\frac{\partial S}{\partial x}$ from (75) and then multiplying the equations by $r\left(p^{+}\right)^{r-1}, r\left(p^{-}\right)^{r-1}$, we can deduce the claim for general $1 \leq r \leq \infty$. Thus we complete a sketch of the proof of the theorem.

\section{B Appendix: Proof of Theorem 3}

Assume $D_{S}=D_{F}=0$, and assume $h, \lambda$ are given by (12), (26), respectively.

(i) If $\gamma=0$ then from (48), $\mathcal{S}^{\prime} \leq 0$ and $\mathcal{S}$ non-increasing in the whole domain, therefore Theorem 3 (i) follows.

(ii) Let us assume $\gamma>0$. We treat $c$ as a parameter, and denote

$$
t_{1}=\frac{\beta}{c \sigma_{1}}=\frac{\beta(s+c)}{2 \lambda_{0} c}, \quad t_{2}=\frac{\beta}{c \sigma_{2}}=\frac{\beta(s-c)}{2 \lambda_{0} c}, \quad \tau_{1}=\frac{\gamma}{c \sigma_{1}}, \quad \tau_{2}=\frac{\gamma}{c \sigma_{2}} .
$$

Solving (49) analytically using (47) and noticing that we assumed the normalization $n_{0}=1$, we obtain (54). Passing to the limits $\xi \rightarrow \pm \infty$, we have $F_{0}=F_{+} e^{-t_{2}}$ and $F_{-}=F_{+} e^{-t_{1}-t_{2}}=F_{+} e^{-\beta s \lambda_{0}^{-1} c^{-1}}$. Therefore the condition $F_{-}>z_{c}$ is equivalent to

$$
F_{+} e^{-\beta / \lambda_{0}-2 \tau_{2} \theta}=F_{+} e^{-\beta s \lambda_{0}^{-1} c^{-1}}>z_{c} .
$$

Using (47), (54), we can solve $\mathcal{S}$ from (48)

$$
S=e^{\frac{\gamma}{c} \xi}\left(S_{0}-\frac{\alpha}{c} \int_{0}^{\xi} \mathcal{F} \mathcal{N} e^{-\frac{\gamma}{c} \xi} \mathrm{d} \xi\right)
$$

or (53). We next show that $\mathcal{S}$ given by (53) is bounded and positive if and only if

$$
I\left(\theta, \tau_{2}\right)=1
$$

which is equivalent to

$$
\tau_{2} e^{t_{2}} t_{2}^{-1-\tau_{2}} \int_{0}^{t_{2}} \eta^{\tau_{2}} e^{-\eta} \mathrm{d} \eta=1
$$

For $\xi \leq 0$, by (53), $S \geq S_{0} e^{\frac{\gamma}{c} \xi}$ and

$$
\begin{aligned}
S & =S_{0} e^{\frac{\gamma}{c} \xi}\left[1+\tau_{1} e^{-t_{1}} t_{1}^{-1+\tau_{1}} \int_{t_{1} e^{\sigma_{1} \xi}}^{t_{1}} \eta^{-\tau_{1}} e^{\eta} \mathrm{d} \eta\right] \\
& \leq S_{0} e^{\frac{\gamma}{c} \xi}\left[1+\tau_{1} e^{-t_{1}} t_{1}^{-1+\tau_{1}}\left(t_{1} e^{\sigma_{1} \xi}\right)^{-\tau_{1}} t_{1} e^{t_{1}}\right] \\
& =S_{0} e^{\frac{\gamma}{c} \xi}\left[1+\tau_{1} e^{-\frac{\gamma}{c} \xi}\right] \leq S_{0}\left(1+\tau_{1}\right)
\end{aligned}
$$


Therefore $\mathcal{S}$ is bounded in $\xi \leq 0$. For $\xi \geq 0$, by (53) and (80),

$$
\begin{aligned}
S & =S_{0} \tau_{2} e^{t_{2}} t_{2}^{-\left(1+\tau_{2}\right)} e^{\frac{\gamma}{c} \xi} \int_{0}^{t_{2} e^{-\sigma_{2} \xi}} \eta^{\tau_{2}} e^{-\eta} \mathrm{d} \eta \\
& =S_{0} \tau_{2} e^{t_{2}} t_{2}^{-\left(1+\tau_{2}\right)} e^{\frac{\gamma}{c} \xi} \bar{\gamma}\left(\tau_{2}+1 ; t_{2} e^{-\sigma_{2} \xi}\right) \\
& =\frac{S_{0} \tau_{2}}{1+\tau_{2}} e^{t_{2}\left(1-e^{-\sigma_{2} \xi}\right)-\sigma_{2} \xi} F_{1}\left(1 ; \tau_{2}+2 ; t_{2} e^{-\sigma_{2} \xi}\right) \\
& =\frac{S_{0} \tau_{2}}{1+\tau_{2}} e^{t_{2}-\sigma_{2} \xi} F_{1}\left(1 ; \tau_{2}+2 ;-t_{2} e^{-\sigma_{2} \xi}\right)
\end{aligned}
$$

where $\bar{\gamma}(a ; x)$ is the lower incomplete gamma function and $F_{1}(a ; b ; x)$ is the confluent hypergeometric function of the first kind (see [1], Chapter 13). Since

$$
\bar{\gamma}\left(\tau_{2}+1 ; t_{2} e^{-\sigma_{2} \xi}\right) \leq \frac{1}{1+\tau_{2}} t_{2}^{1+\tau_{2}} e^{-\sigma_{2}\left(1+\tau_{2}\right) \xi},
$$

we have

$$
S \leq S_{0} \tau_{2} e^{t_{2}} t_{2}^{-\left(1+\tau_{2}\right)} e^{\frac{\gamma}{c} \xi} \frac{1}{1+\tau_{2}} t_{2}^{1+\tau_{2}} e^{-\sigma_{2}\left(1+\tau_{2}\right) \xi}=\frac{S_{0} \tau_{2}}{1+\tau_{2}} e^{t_{2}},
$$

with equality holding only when $\xi=0$. Since $\lim _{\xi \rightarrow+\infty} F_{1}\left(1 ; \tau_{2}+2 ; t_{2} e^{-\sigma_{2} \xi}\right)=F_{1}\left(1 ; \tau_{2}+2 ; 0\right)=1$, we have $\lim _{\xi \rightarrow+\infty} S=0$. Therefore $\mathcal{S}$ is bounded in $\xi>0$. Assume (80) is violated, direct calculation shows that $\mathcal{S}$ is unbounded.

It is easy to see that

$$
I(\theta, 0)=0 .
$$

As $z \rightarrow \infty$, by Stirling's formula, we have

$$
I(\theta, z) \sim \frac{e^{\theta z} z !}{\theta^{1+z} z^{z}} \sim \frac{e^{\theta z} \sqrt{2 \pi z}(z / e)^{z}}{\theta^{1+z} z^{z}}=\frac{\sqrt{2 \pi z}}{\theta} \exp [(\theta-1-\ln \theta) z] .
$$

Since $\theta-1-\ln \theta \geq 0$, we obtain that $\lim _{z \rightarrow \infty} I(\theta, z)=\infty$. Therefore there exist solutions to (79).

We next show that under (79), then $\mathcal{S}$ given by (53) satisfies $\mathcal{S} \in Y_{S}$ if and only if

$$
t_{2} \geq 1 \Leftrightarrow \tau_{2} \geq \frac{1}{\theta} .
$$

Differentiating (48) once we obtain,

$$
\mathcal{S}^{\prime \prime}=\left\{\begin{array}{cc}
-\frac{\alpha \sigma_{1}}{c} \mathcal{F} \mathcal{N}\left(t_{1} \mathcal{N}+1\right)+\frac{\gamma}{c} \mathcal{S}^{\prime}, & \xi<0 \\
-\frac{\alpha \sigma_{2}}{c} \mathcal{F} \mathcal{N}\left(t_{2} \mathcal{N}-1\right)+\frac{\gamma}{c} \mathcal{S}^{\prime}, & \xi>0 .
\end{array}\right.
$$

At local extrema, we have $\mathcal{S}^{\prime}=0$ and

$$
\mathcal{S}^{\prime \prime}= \begin{cases}-\frac{\alpha \sigma_{1}}{c} \mathcal{F} \mathcal{N}\left(t_{1} \mathcal{N}+1\right), & \xi<0 \\ -\frac{\alpha \sigma_{2}}{c} \mathcal{F} \mathcal{N}\left(t_{2} \mathcal{N}-1\right), & \xi>0 .\end{cases}
$$

For $\mathcal{S} \in Y_{S}, \xi=0$ should be a global maximum. Since $\mathcal{S}^{\prime}(0)=0$, it requires $\mathcal{S}^{\prime \prime}(0-) \leq 0$ and $\mathcal{S}^{\prime \prime}(0+) \leq 0$. From (83), $\mathcal{S}^{\prime \prime}(0-)=-\alpha \sigma_{1} c^{-1} F_{0}\left(t_{1}+1\right)<0$, and $\mathcal{S}^{\prime \prime}(0+)=-\alpha \sigma_{2} c^{-1} F_{0}\left(t_{2}-1\right) \leq 0$ if and only if (82). Assume (82), we prove that $\mathcal{S}^{\prime}(\xi) \neq 0$ given $\xi \neq 0$, i.e., $\mathcal{S}$ has no other finite extrema. Therefore since $\mathcal{S}^{\prime}$ is continuous, $\mathcal{S}^{\prime}>0$ for $\xi<0$ and $\mathcal{S}^{\prime}<0$ for $\xi>0$. Assume $\mathcal{S}$ has a local minimum or saddle point $\xi_{0}<0$, then $\mathcal{S}^{\prime \prime}\left(\xi_{0}\right) \geq 0$, but from (83) $\mathcal{S}^{\prime \prime}\left(\xi_{0}\right)<0$ which leads to contradiction. Assume $\xi_{0}<0$ is a local maximum, since $\mathcal{S}^{\prime \prime}\left(\xi_{0}\right)<0, \mathcal{S}^{\prime \prime}(0-)<0$, we can find $\xi_{0}<\xi_{1}<\xi_{2}<0$, such that $\mathcal{S}^{\prime}\left(\xi_{1}\right)<0<\mathcal{S}^{\prime}\left(\xi_{2}\right)$, therefore there exists $\xi_{3} \in\left(\xi_{1}, \xi_{2}\right)$ such that $\mathcal{S}^{\prime}\left(\xi_{3}\right)=0$ and $\mathcal{S}^{\prime \prime}\left(\xi_{3}\right) \geq 0$, again a contradiction. A similar argument leads to 
$\mathcal{S}^{\prime}<0$ in $\xi \in\left(0, \xi_{*}\right)$ with $\xi_{*}=\log \left(t_{2}\right) \sigma_{2}^{-1}, N\left(\xi_{*}\right)=\frac{1}{t_{2}}$. For $\xi>\xi_{*}$ since $\mathcal{S}^{\prime \prime}>0$, no local maximum or saddle point is possible. Assume $\xi_{1}>\xi_{*}$ is a local minimum, then $\mathcal{S}^{\prime}>0$ for all $\xi>\xi_{1}$ and $\mathcal{S}\left(\xi_{1}\right)<0=\lim _{x \rightarrow+\infty} \mathcal{S}$, a contradiction. For $\xi=\xi_{*}, \mathcal{S}^{\prime} \neq 0$ by using $(48,81, \mathrm{~B})$.

We now prove (ii) of the theorem. If there exists non-starved traveling wave solutions with $\min F=F_{-}>$ $z_{c}$ and $\mathcal{S} \in Y_{S}$, then by the above arguments $\mathcal{N}, \mathcal{S}, \mathcal{F}$ are given by (47), (53) and (54) and satisfy (78), (79), and (82). On the other hand, if there exists $\tau_{2}$ that satisfies (78), (79), and (82), then the functions such that $\mathcal{N}, \mathcal{S}, \mathcal{F}$ given by (47), (53) give a traveling wave solution of the system (28)-(33) with $c$ given by (52).

A sufficient condition for the existence of $\tau_{2}$ that satisfies both (79) and (82) is

$$
I\left(\theta, \frac{1}{\theta}\right)=\frac{e}{\theta} \int_{0}^{1} \eta^{\frac{1}{\theta}} e^{-\eta} \mathrm{d} \eta<1 .
$$

We show numerically in Figure (7)

$$
I\left(\theta, \frac{1}{\theta}\right)<1, \quad \text { for } \theta>\text { a small positive number, }
$$

This means that when secretion of attractant is not too slow compared to $\gamma$, the system (28)-(33) has a traveling wave solution.

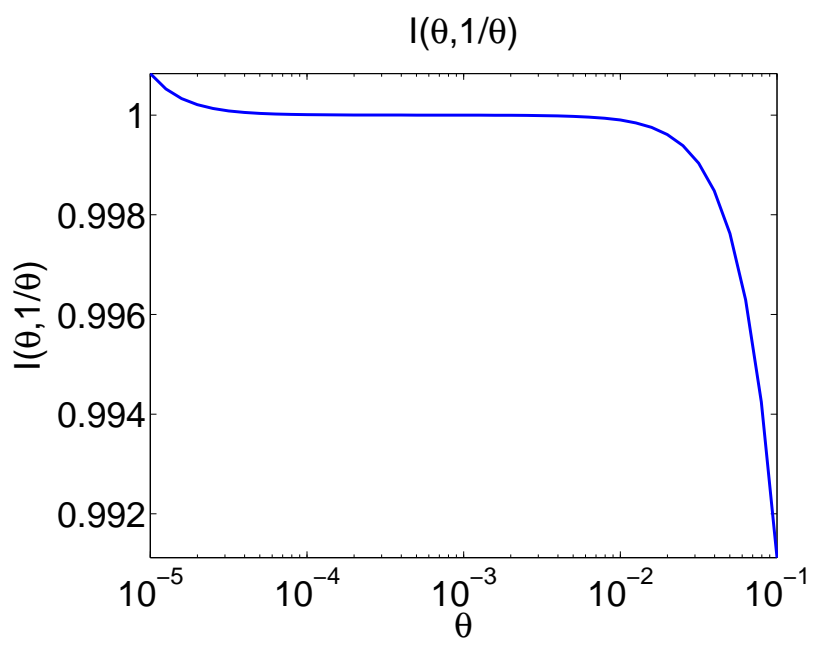

Fig. 7 Numerical approximation of $I\left(\theta, \frac{1}{\theta}\right)$. Trapezoidal rule with $h=10^{-6}$ is used to evaluate the integral.

\section{Appendix: Proof of Theorem 4}

By Picard's Theorem the solution of (45), (46), (43), (44) and (57) with initial conditions at $\xi=0$ exists locally at every point in the phase space. In addition solutions can be extended to $\xi \in \mathbb{R}$ and $\mathcal{F}$ is monotonically increasing. We show below that there exists $\mathcal{N}_{z}(0), \mathcal{J}_{z}(0)$ and $\mathcal{F}(0)$ such that $\mathcal{N}_{z}, \mathcal{J}_{z}$ and $\mathcal{F}$ are bounded and satisfy the boundary conditions (40).

Denote the normed space

$$
Y=\left\{f ; f, f^{\prime} \in C(\mathbb{R}) \cap L^{\infty}(\mathbb{R})\right\} \quad \text { with } \quad\|f\|_{Y}=\sup _{x \in \mathbb{R}}|f(x)|+\sup _{x \in \mathbb{R}}\left|f^{\prime}(x)\right| .
$$

One can easily show that $Y$ is a Banach space by proving its completeness using standard real analysis techniques. Denote its closed subset

$$
Y_{F}=\{f \in Y ; f \text { non-decreasing }\} .
$$


For $\mathcal{F} \in Y_{F}$, we solve the equations (44) and (46) for $m$ and give conditions on $m_{0}=m(0)$ such that $m(\xi) \rightarrow 0$ as $\xi \rightarrow \pm \infty$. We first consider the interval $\xi>0$. The eigenvalues and corresponding eigenvectors of $A$ are

$$
\begin{array}{rlrl}
\mu_{1} & =\frac{2 \lambda_{0}+t_{m}^{-1}}{s-c}>0, & u_{1} & =\left(\begin{array}{c}
2 \lambda_{0}+t_{m}^{-1} \\
2 \lambda_{0} c+s t_{m}^{-1}
\end{array}\right), \\
\mu_{2}=-\frac{1}{t_{m}(s+c)}<0, & u_{2}=\left(\begin{array}{c}
1 \\
-s
\end{array}\right) .
\end{array}
$$

The vector $a$ can be decomposed into a linear combination of the eigenvectors

$$
a=c_{1} u_{1}+c_{2} u_{2}, \quad \text { with }\left\{\begin{array}{l}
c_{1}=\frac{s+c}{2(s-c)\left(\lambda_{0} t_{m}(s+c)+s\right)}>0, \\
c_{2}=-\frac{2 \lambda_{0}(s+c)+(s-c) t_{m}^{-1}}{2(s+c)\left(\lambda_{0} t_{m}(s+c)+s\right)}<0 .
\end{array}\right.
$$

Assuming $m_{0}=q_{10} u_{1}+q_{20} u_{2}$, then the solution of $m$ satisfies

$$
m=q_{1}(\xi) u_{1}+q_{2}(\xi) u_{2} \quad \text { for } \xi \geq 0
$$

with

$$
q_{1}(\xi)=e^{-\mu_{1} \xi}\left[q_{10}+c_{1} \int_{0}^{\xi}(\mathcal{F N})(\zeta) e^{\mu_{1} \zeta} \mathrm{d} \zeta\right], \quad q_{2}(\xi)=e^{-\mu_{2} \xi}\left[q_{20}+c_{2} \int_{0}^{\xi}(\mathcal{F N})(\zeta) e^{\mu_{2} \zeta} \mathrm{d} \zeta\right]
$$

From the above formulae we obtain that, $q_{1} \rightarrow 0$ as $\xi \rightarrow \infty$ for any $q_{10}$. For $q_{2} \rightarrow 0$, by considering the form of $\mathcal{N}$, it is necessary that

$$
q_{20}=-c_{2} \int_{0}^{\infty}(\mathcal{F N})(\zeta) e^{\mu_{2} \zeta} \mathrm{d} \zeta
$$

With this condition we have

$$
q_{2}(\xi)=-c_{2} e^{-\mu_{2} \xi} \int_{\xi}^{+\infty}(\mathcal{F} \mathcal{N})(\zeta) e^{\mu_{2} \zeta} \mathrm{d} \zeta
$$

Similarly, for $\xi<0$, the eigenvalues and corresponding eigenvectors of $B$ are

$$
\begin{aligned}
\gamma_{1} & =\frac{2 \lambda_{0}+t_{m}^{-1}}{s+c}>0, & w_{1} & =\left(\begin{array}{c}
2 \lambda_{0}+t_{m}^{-1} \\
2 \lambda_{0} c-s t_{m}^{-1}
\end{array}\right), \\
\gamma_{2} & =-\frac{1}{t_{m}(s-c)}<0, & w_{2} & =\left(\begin{array}{l}
1 \\
s
\end{array}\right) .
\end{aligned}
$$

The vector a can be decomposed as

$$
a=d_{1} w_{1}+d_{2} w_{2}, \quad \text { with }\left\{\begin{array}{l}
d_{1}=-\frac{s-c}{2(s+c)\left(\lambda_{0} t_{m}(s-c)+s\right)}<0 \\
d_{2}=\frac{2 \lambda_{0}(s-c)+(s+c) t_{m}^{-1}}{2(s-c)\left(\lambda_{0} t_{m}(s-c)+s\right)}>0
\end{array}\right.
$$

Assume $m_{0}=r_{10} w_{1}+r_{20} w_{2}$, then the solution of $m$ satisfies

$$
m=r_{1}(\xi) w_{1}+r_{2}(\xi) w_{2} \quad \text { for } \xi \leq 0
$$

with

$$
r_{1}(\xi)=e^{\gamma_{1} \xi}\left[r_{10}-d_{1} \int_{\xi}^{0}(\mathcal{F} \mathcal{N})(\zeta) e^{-\gamma_{1} \zeta} \mathrm{d} \zeta\right], \quad r_{2}(\xi)=e^{\gamma_{2} \xi}\left[r_{20}-d_{2} \int_{\xi}^{0}(\mathcal{F N})(\zeta) e^{-\gamma_{2} \zeta} \mathrm{d} \zeta\right]
$$


Notice that $r_{1} \rightarrow 0$ as $\xi \rightarrow-\infty$ given $\mathcal{F} \in Y_{F}$. However for $r_{2} \rightarrow 0$, it is required that

$$
r_{20}=d_{2} \int_{-\infty}^{0}(\mathcal{F} \mathcal{N})(\zeta) e^{-\gamma_{2} \zeta} \mathrm{d} \zeta
$$

which is well-defined for bounded $\mathcal{F}$. Under this condition we have

$$
r_{2}(\xi)=d_{2} e^{\gamma_{2} \xi} \int_{-\infty}^{\xi}(\mathcal{F N})(\zeta) e^{-\gamma_{2} \zeta} \mathrm{d} \zeta
$$

For $m(\xi) \rightarrow 0$ as $\xi \rightarrow \pm \infty$, the value $m_{0}$ should satisfy

$$
m_{0}=\left(\begin{array}{c}
r_{20}\left(1+\lambda_{0} t_{m} \frac{s-c}{s}\right)+q_{20}\left(1+\lambda_{0} t_{m} \frac{s+c}{s}\right) \\
\frac{r_{20}\left(2 \lambda_{0} c+s t_{m}^{-1}\right)\left(\lambda_{0}(s-c) t_{m} s^{-1}+1\right)+q_{20}\left(2 \lambda_{0} c-s t_{m}^{-1}\right)\left(\lambda_{0}(s+c) t_{m} s^{-1}+1\right)}{2 \lambda_{0}+t_{m}^{-1}}
\end{array}\right),
$$

with the decomposition coefficients $q_{20}$ and $r_{20}$ given by (86) and (90), and $q_{10}$ and $r_{10}$ can be calculated as

$$
q_{10}=\frac{r_{20}\left[\lambda_{0} t_{m}(s-c) / s+1\right]+q_{20} \lambda_{0} t_{m}(s+c) / s}{2 \lambda_{0}+t_{m}^{-1}}, \quad r_{10}=\frac{r_{20} \lambda_{0} t_{m}(s-c) / s+q_{20}\left[\lambda_{0} t_{m}(s+c) / s+1\right]}{2 \lambda_{0}+t_{m}^{-1}}
$$

Combining the above analysis, we obtain the following lemma:

Lemma 5 Given $\mathcal{F} \in Y_{F}$, the solution of (44), (46) has the form (84), (88) with (85), (87), (89), (91). For $m(\xi) \rightarrow 0$ as $\xi \rightarrow \pm \infty$, the value $m_{0}$ should satisfy (92) with (86), (90) and (93).

Notice that the case when $m$ is not bounded is biologically relevant to the model, therefore we do not consider here. Set

$$
Y_{m}=\left\{(n, j) ; n, j \in C(\mathbb{R}) \cap L^{\infty}(\mathbb{R})\right\} \quad \text { with } \quad\|(n, j)\|_{Y_{m}}=\sup _{x \in \mathbb{R}}|n|+\sup _{x \in \mathbb{R}}|j| .
$$

Now define the mapping $W_{1}: Y_{F} \rightarrow Y_{m}$ that maps $\mathcal{F} \in Y_{F}$ to the solution of (44), (46) as a function of $\mathcal{F}$ with (92). We also define the mapping $W_{2}: Y_{m} \rightarrow Y_{F}$ by

$$
W_{2}(m)=F_{0} \exp \left[\int_{0}^{\xi} \frac{\beta}{c} \mathcal{N}_{z} \mathrm{~d} \zeta\right]
$$

therefore the equation (57) for $\mathcal{F}$ is equivalent to

$$
\mathcal{F}=W_{2}(m)
$$

Define the mapping $W=W_{2} W_{1}$, then we can prove that $W$ maps $Y_{F}$ to $Y_{F}$. Indeed, for $\mathcal{F} \leq \bar{F}$, we can get estimates of the $q_{i}(\xi), r_{i}(\xi)$. From these estimations we obtain $\mathcal{N}_{z} \leq \bar{F} \mathcal{N}$, and therefore using (47) and (94) we conclude that $W: Y_{F} \rightarrow Y_{F}$. Using the formulae referred in Lemma 5 and (95), by direct calculation we can show that the mapping $W$ is a contraction. Since the computations are straightforward, we omit the details. By the contraction mapping theorem, there exists a fixed point of this mapping, which proves the first half of Theorem 4 .

The following lemma proves the second half of the theorem.

\section{Lemma 6 For}

$$
F_{0} \exp \left[\frac{\beta(s-c)}{2 \lambda_{0} c}\right]=F_{0} e^{t_{2}} \leq 1
$$

we have $F_{+}=\lim _{\xi \rightarrow+\infty} \mathcal{F}(\xi) \leq 1$.

Proof. Assume $[0, M]$ is the largest interval for $\mathcal{F} \leq 1$. Then in this interval, we have $\mathcal{N}_{z} \leq \mathcal{N}$. By comparison theorem, $\mathcal{F}(M) \leq F_{0} e^{t_{2}\left(1-e^{-\sigma_{2} M}\right)}<1$. Therefore the solution satisfies $\mathcal{F} \leq 1$ in $[M, M+\epsilon]$. Contradiction. Therefore $\mathcal{F} \leq F_{+} \leq 1$. 


\section{References}

1. M. Abramowitz and I.A. Stegun, Handbook of mathematical functions with formulars, graphs, and mathematical tables, United States Government Printing Office, 9th edtion, 1964.

2. J. Adler, Chemotaxis in bacteria, Science 153 (1966), 708-716.

3. J. Adler, Effect of amino acids and oxygen on chemotaxis in escherichia coli, Journal of Bacteriology 92 (1966), 121-129.

4. Chemotaxis in bacteria, Annual Reviews Biochemistry 44 (1975), 341-356.

5. N. Barkai and S. Leibler, Robustness in simple biochemical networks, Nature 387 (1997), 913-917.

6. N. Bournaveas, A. Buguin, V. Calvez, B. Perthame, J. Saragosti and P. Silberzan, Mathematical description of bacterial traveling pulses, under revision (2010).

7. N. Bournaveas and V. Calvez, Global existence for the kinetic chemotaxis model without pointwise memory effects, and including internal variables, Kinetic and Related Models 1 (2008), no. 1, 29-48.

8. M. Brenner, L. Levitov, and E. Budrene, Physical mechanisms for chemotactic pattern formation by bacteria, Biophysical J. 74 (1998), no. 4, 1677-1693.

9. E. O. Budrene and H. C. Berg, Complex patterns formed by motile cells of escherichia coli, Nature 349 (1991), no. $6310,630-633$.

10. Dynamics of formation of symmetrical patterns by chemotactic bacteria, Nature 376 (1995), no. 6535, 49-53.

11. F. Dahlquist, P. Lovely, and D. Koshland, Quantitative analysis of bacterial migration in chemotaxis, Nature New Biology 236 (1972), 120-123.

12. R. Erban, From individual to collective behaviour in biological systems, Ph.D. thesis, University of Minnesota, 2005.

13. R. Erban and H. Hwang, Global existence results for the complex hyperbolic models of bacterial chemotaxis, Discrete and Continuous Dynamical Systems Series B 6 (2006), no. 6, 1239-1260.

14. R. Erban and H. Othmer, From individual to collective behaviour in bacterial chemotaxis, SIAM Journal on Applied Mathematics 65 (2004), no. 2, 361-391.

15. - From signal transduction to spatial pattern formation in E. coli: A paradigm for multi-scale modeling in biology, Multiscale Modeling and Simulation 3 (2005), no. 2, 362-394.

16. R. Erban and H. G. Othmer, Taxis equations for amoeboid cells, J. Math. Biol. 54 (2007), 847-885.

17. A. Gerisch and K.J. Painter, Mathematical modelling of cell adhesion and its applications to developmental biology and cancer invasion., Submitted (2009).

18. D. Horstmann, From 1970 until present: the Keller-Segel model in chemotaxis and its consequences I, Jahresbericht der DMV 105 (2003), no. 3, 103-165.

19. D. Horstmann and A. Stevens, A constructive approach to traveling waves in chemotaxis, Journal of Nonlinear Science 14 (2004), 1-25(25).

20. H. Hwang, K. Kang, and A. Stevens, Drift-diffusion limits of kinetic models for chemotaxis: a generalization, Discrete and Continuous Dynamical Systems B 5 (2005), no. 2, 319-334.

21. __ Global solutions of nonlinear transport equations for chemosensitive movements, SIAM Journal on Mathematical Analysis 36 (2005), no. 4, 1177-1199.

22. _ Global existence of classical solutions for a hyperbolic chemotaxis model and its parabolic limit, Indiana University Mathematics Journal 55 (2006), no. 1, 289-316.

23. E. F. Keller and L. A. Segel, Traveling bands of chemotactic bacteria: A theoretical analysis, J. Theor. Biol. 30 (1971), 235-248.

24. K. Landman, G. Petter, and D. Newgreen, Chemotactic cellular migration: smooth and discontinuous travelling wave solutions, SIAM Journal on Applied Mathematics 63 (2003), no. 5, 1666-1681.

25. D. Lauffenburger, C. R. Kennedy, and R. Aris, Traveling bands of chemotactic bacteria in the context of population growth, Bulletin of Mathematical Biology 46 (1984), no. 1, 19-40.

26. T. Li and Z. Wang, Nonlinear stability of large amplitude viscous shock waves of a hyperbolic-parabolic system arising in chemotaxis, Mathematical Models and Methods in Applied Sciences, to appear.

27. R. Lui and Z. A. Wang, Traveling wave solutions from microscopic to macroscopic chemotaxis models, J. Math. Biol., in press.

28. J. Murray, Mathematical Biology, Springer Verlag, 2002.

29. K.J. Painter, Modelling cell migration strategies in the extracellular matrix., J. Math. Biol. 58 (2009), no. $511-543$.

30. J. Simon and P. A. Milewski, The volcano effect in bacterial chemotaxis, Mathematical and computer Modeling, in press.

31. P. Spiro, J Parkinson, and H. Othmer, A model of excitation and adaptation in bacterial chemotaxis, Proceedings of the National Academy of Sciences USA 94 (1997), 7263-7268. 
32. B. A. Schmitt, R. Weiner and H. Podhaisky, ROWMAP-a ROW-code with Krylov techniques for large stiff ODEs, Appl. Numer. Math. 25 (1997), 303-319.

33. R. Weis and D. Koshland, Reversible receptor methylation is essential for normal chemotaxis of Escherichia coli in gradients of aspartic acid, PNAS 85 (1988), 83-87.

34. C. Xue and H. G. Othmer, Multiscale models of taxis-driven patterning in bacterial populations, SIAM J. Appl. Math. 70 (2009), no. 1, 133-167.

35. C. Xue, H. G. Othmer, and R. Erban, From individual to collective behavior of unicellular organisms: Recent results and open problems, vol. 1167, AIP, 2009, pp. 3-14. 


\section{RECENT REPORTS}

39/09 Spatially structured oscillations in a two-dimensional excitatory

Kilpatrick neuronal network with synaptic depression

Bressloff

40/09 Stationary bumps in a piecewise smooth neural field model with synaptic depression

Kilpatrick

Bressloff

41/09 Homogenization for advection-diffusion in a perforated domain Haynes

Hoang

Norris

Zygalakis

42/09 Fast stochastic simulation of biochemical reaction systems by alternative formulations of the Chemical Langevin Equation

Melykuti

Burrage

Zygalakis

43/09 Pseudoreplication invalidates the results of many neuroscientific

Lazic studies

44/09 Cardiac cell modelling: Observations from the heart of the cardiac physiome project

45/09 A Hybrid Radial Basis Function - Pseudospectral Method for Thermal Convection in a 3-D Spherical Shell

Wright

Flyer

46/09 Refining self-propelled particle models for collective behaviour

Yates

Baker

Erban

Maini

47/09 Stochastic Partial Differential Equations as priors in ensemble methods for solving inverse problems

Potsepaev

Farmer

Aziz

48/09 DifFUZZY: A fuzzy spectral clustering algorithm for complex data

Cominetti et al. sets

01/10 Fluctuations and instability in sedimentation

Guazzelli

Hinch

02/10 Determining the equation of state of highly plasticised metals from

Hinch boundary velocimetry

03/10 Stability of bumps in piecewise smooth neural

Kilpatrick elds with nonlinear adaptation

Bressloff

04/10 Random intermittent search and the tug-of-war model of motordriven transport

Newby

Bressloff

05/10 Ergodic directional switching in mobile insect groups

Escudero et al. 
06/10 Derivation of a dual porosity model for the uptake of nutrients by root hairs

Zygalakis

Roose

07/10 Frost heave in compressible soils

Majumdar

Peppin

Style

Sander

08/10 A volume-preserving sharpening approach for the propagation of

Reis sharp phase boundaries in multiphase lattice Boltzmann simulations

Dellar

09/10 Anticavitation and differential growth in elastic shells

Moulton

Goriely

10/10 On the mechanical stability of growing arteries

Goriely

Vandiver

11/10 Nonlinear Correction to the Euler Buckling Formula for Compressible Cylinders

De Pascalis

Destrade

Goriely

12/10 Nonlinear Morphoelastic Plates I: Genesis of Residual Stress

McMahon

Goriely

Tabor

13/10 Nonlinear Morphoelastic Plates II: Exodus to Buckled States

McMahon

Goriely

Tabor

14/10 Analysis of Brownian dynamics simulations of reversible biomolec-

Lipkova ular reactions

Zygalakis

Chapman

Erban

Copies of these, and any other OCCAM reports can be obtained from:

Oxford Centre for Collaborative Applied Mathematics Mathematical Institute

24 - 29 St Giles'

Oxford

OX1 3LB

England

www.maths.ox.ac.uk/occam 